\title{
The WTE+ Framework: Automated Construction and Runtime Adaptation of Service Mashups
}

\author{
Anna Hristoskova • Bruno Volckaert • \\ Filip De Turck
}

Received: date / Accepted: date

\begin{abstract}
An emerging software engineering methodology is the combination of functionality and content from existing software components into service mashups, creating greater value than the sum of the individual participating building blocks. For businesses, using catalogues of reusable services means agile development of new applications using open communication standards including the Simple Object Access Protocol (SOAP) for transmitting data, and the Web Services Description Language (WSDL) for defining services. The result is faster adaptation to the changing business environment creating value at reduced development time and cost while increasing revenues.

This manuscript presents the developed service management framework and evaluation results of the WTE+ (Web2.0/Telco2.0/Enterprise2.0 with semantics) research project. Main goal is supporting application developers with the automatic construction and runtime reconfiguration of custom-made service mashups at a minimum performance cost without the need for constant IT intervention. Semantically enriched services are automatically combined into custom-made service mashups by the designed planning algorithms. These planning techniques are optimized with late binding and runtime adaptation to changing user-context taking fully into account the quality of service parameters of the available components which are provided at design time and updated at runtime. In addition, recovery mechanisms are provided in case of
\end{abstract}

\footnotetext{
A. Hristoskova · B. Volckaert · F. De Turck

Department of Information Technology, Ghent University - IBBT

Gaston Crommenlaan 8 bus 201, B-9050 Ghent, Belgium

Tel.: +32-9-33-14974

Fax: +32-9-33-14899

E-mail: Anna.Hristoskova@intec.UGent.be

B. Volckaert

E-mail: Bruno.Volckaert@intec.UGent.be

F. De Turck

E-mail: Filip.DeTurck@intec.UGent.be
} 
failed services and/or resources. During mashup execution, unavailable services are dynamically replaced by equivalent ones or alternative service mashups keeping in mind the current execution state.

The developed planning algorithms are put through extensive performance and scalability experiments for a typical e-commerce scenario, in which e-shop services such as product payment and delivery are on-the-fly composed to an e-shop application. The results show that an automatic construction of a new application out of existing services can take up between 5 to 43 seconds for 500 services while runtime adaptation takes up to 5 seconds on average depending on the availability of equivalent services.

Keywords Service mashups · Semantic reasoning · HTN planning · Runtime adaptation · Quality of Service (QoS)

\section{Introduction}

Nowadays everybody is connected using a broadband connection at home or at work, a workstation at school or a mobile device. Since the Web 2.0 hype, Web sites have changed from static pages to interactive applications enabling the sharing of content and services. Several distinct trends (Hausheer et al 2009) are observed in the evolution of the Internet that will transform the business models of industry and the way software is developed.

In the first place as more companies find their way to the Internet and the necessary technologies are available for an ever wider audience, the number of companies (and even governments) that offers online services increases steadily. The result is a demand for software applications aiming to find and use the best combination of services - service mashup - on the Web fulfilling a particular task (Taivalsaari and Mikkonen 2008). A service mashup is a new service that combines functionality or content from existing sources. These sources can be Web services, software components capable of being accessed via standard network protocols such as but not limited to SOAP over HTTP. The mashup construction is realized due to a workflow description: definition of how the basic building blocks interact. Today there are a number of popular standards and implementations such as BPMN, WFF, BPEL4WS, XLANG, WSFL which define workflows (Peltz 2003; Van Der Aalst et al 2003). However they still exhibit a number of shortcomings: no clearly defined semantics, no automatic or dynamic deployment support, limited reliability guarantees.

A second trend is the offered service diversity. Ambient intelligence environments enable universal and immediate access to the available resources. Cars are able to schedule maintenance when required and seamlessly navigate through the everyday traffic providing the most suited context information to their passengers. As such applications consist of multiple collaborating services the number of dependencies will increase, thus increasing the chance of failure. These smart devices should be able to communicate fluently with each other realizing dynamic, self-adaptive systems (Di Nitto et al 2008). 
A third important trend for the Web 2.0 as well as for business applications is that the programmer no longer develops the end-application, but only provides the end-user with the necessary tools to put together his own custom-made applications. This manifests itself both with the proliferation of mashups on the Internet often created by end-users and at companies where domain experts closely collaborate with the IT department in order to exactly design the required applications. However, a common rule of thumb in software engineering is that since development of services takes several weeks, their integration can take the same amount of time. This negotiating of requirements, planning, implementation, testing and deployment process should be reduced, leaving all parties to do their respective jobs more optimally. By introducing an application management framework, which is able to automatically assemble new service mashups, businesses will no longer need to contact developers each time new functionality is required. In a dynamic environment where the desired functionality cannot always be predicted, all kinds of custom-made mashups can be built from scratch to meet the users' needs.

These trends confirm the need to dynamically combine and adapt the growing amount of diverse services, tackle failure points due to service dependencies and still be able to provide in a transparent manner personalized applications to the end-user. The WTE+ project (WTE+ 2008-2010) tackles those issues by defining the extension of Web2.0/ Telco2.0/ Enterprise 2.0 with semantics, reasoning, autonomous operation and distributed data repository. The project consortium consists of several partners such as service information providers, telecom operators, e-commerce, and medical imaging companies. The core objective is the definition of an open architecture usable over a broad range of industries that allows the easy creation of new service mashups departing from available software components. An important aspect is the enrichment of the service composition with the use of semantic technologies, further increasing the level of abstraction while creating opportunities to select the most appropriate function providers at runtime.

The developed WTE + management framework, detailed in this paper, offers an environment for the automatic construction and execution of service mashups departing from available functionality found on the Web and within enterprises. This approach is substantiated by the following novel contributions:

- User interface managing the semantically annotated services, their QoS parameters and users' requests.

- Planning algorithms automatically constructing service mashups using existing semantically enriched services adopting the richness of the semantic language OWL-S which supports workflow characteristics such as control constructs ('Split+Join', 'IfThenElse', 'ForEach', 'Choice').

- Automatic addition of control constructs during the composition process depending on the quality of the match between the individual services resulting in service workflows. 
- Dynamic adaptation to changing runtime environment through the monitoring and updating of service QoS parameters.

- Partial workflow execution optimizing the constructed mashup by feeding back intermediary results used for its reconfiguration.

- Request personalization through user-defined business logic rules.

- Plug-in design of the overall framework allowing for future additions of new QoS-aware planning algorithms and execution engines.

The developed methods are validated by an e-commerce scenario involving one of the industry partners of the WTE+ project where focus lies on providing a high level interface to third party services used to create new applications. In this context existing e-shop services such as product selection, ordering, payment and delivery are semantically annotated and on-the-fly composed into an e-shop application.

The remainder of this paper is structured as follows: Section 2 presents the general concept and functional requirements of the WTE+ management framework. An overview of the current state of the art and available technologies concerning application management is given in Section 3. Section 4 elaborates on the architecture of the mashup creation and execution framework whose implementation is detailed in Section 5. The performance and scalability measurements are analyzed in Section 6 for an e-shop application. Finally, the main conclusions are drawn in Section 7.

\section{General Concept}

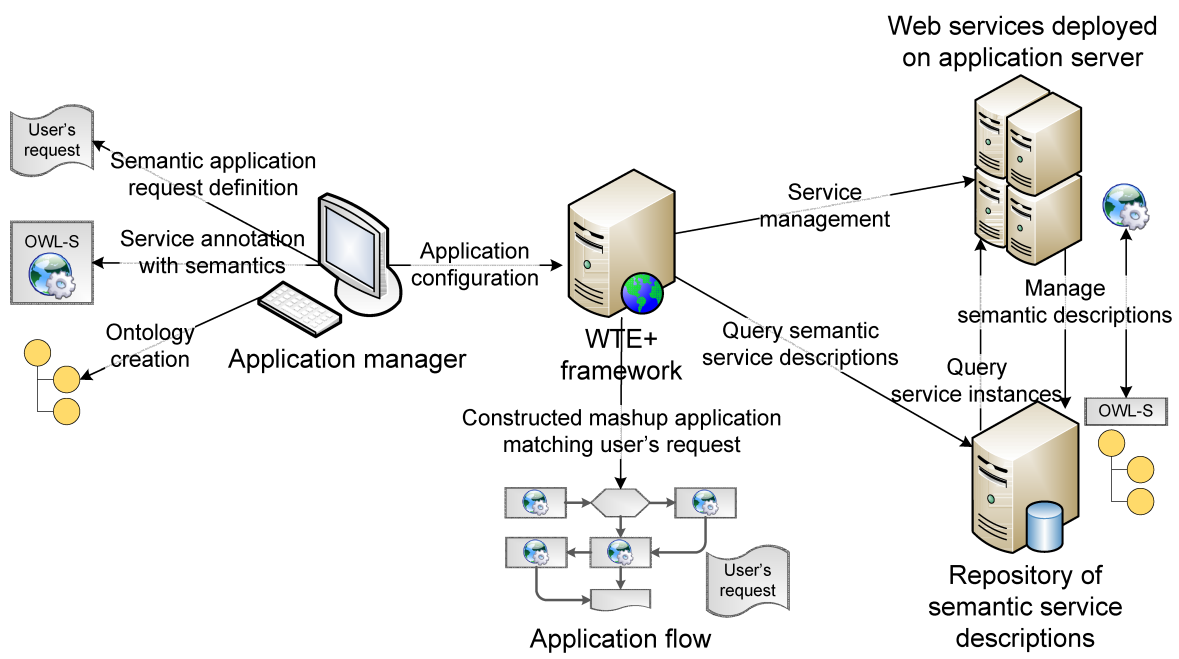

Fig. 1 Configuration of the WTE+ management framework consisting of an ontology definition, service enrichment with semantics and new mashup creation based on a defined request. 


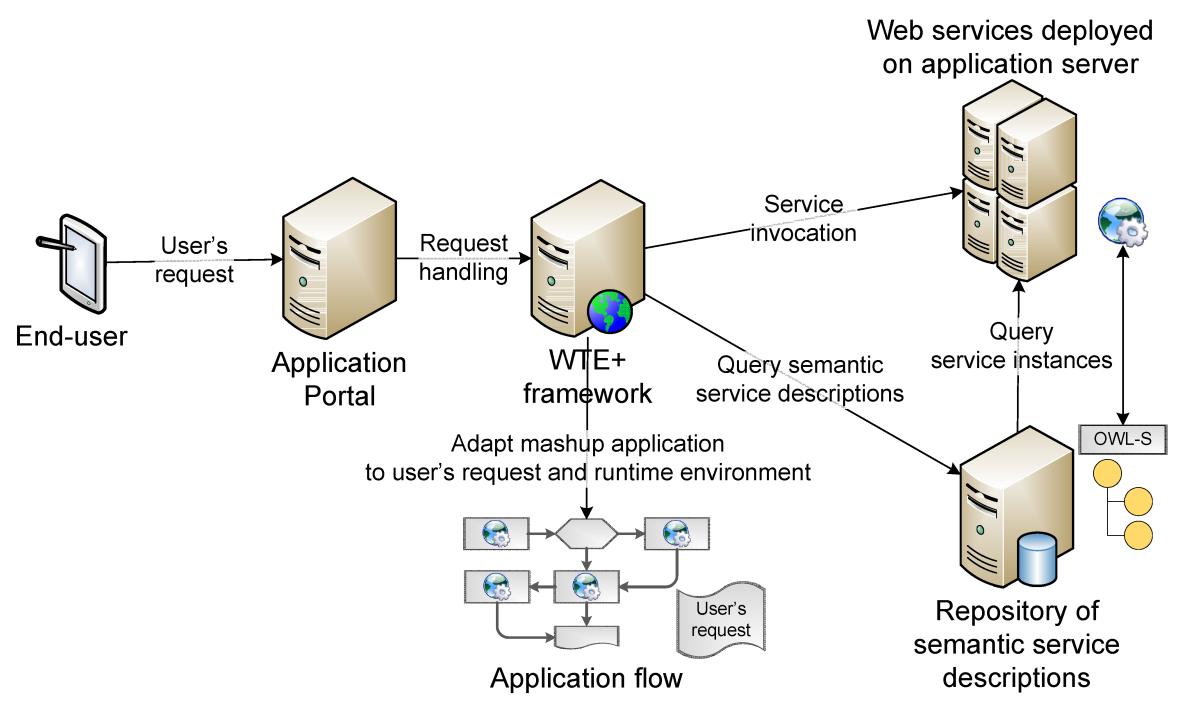

Fig. 2 General concept of the use of the WTE+ management framework by and end-user.

The main objective of this research is the design of a management framework supporting the development lifecycle of new applications by assembling available functionality found on the Web and within enterprises with minimal intervention by the ICT department. Figure 1 presents an overview of the use of the framework by a developer as the actual administrator of the system, responsible for the management of the available software components within the enterprise, the definition of their quality parameters, and the construction and configuration of the required user-application. Requirement is some amount of knowledge on the use of ontologies and semantic description of services. There are several steps that should be considered. First one should start with a definition of an ontology. Depending on the application at hand several existing ontologies can be found on the web or a new one can be defined dedicated for internal use within the enterprise environment. Using the ontology the existing enterprise services should be semantically annotated. One can use existing automatic annotation techniques such as the OWL-S API by (Möller 2010) where the WSDL service description is automatically enriched and tuned to the specific ontology. Once this process is executed correctly, these ontologies and services can be reused and shared between company boundaries. A third step consists of the specification of a user's requests. One can define several goals useful for the company environment. After that the developer can proceed with the use of the WTE+ framework. The semantic service descriptions are registered within the framework which automatically uploads the necessary ontological files and references to service instances. Selecting a user request results in the automatic construction of a new service out of the existing services. The framework provides a graphical user interface in Section 4.1 where one can tune the constructed mashup to his needs by trying out dif- 
ferent services and test the end-application. The result is a fast development of a new application based on the available enterprise resources.

For the actual end-use execution of the new application, we assume a tailored portal is available as an interface to the WTE+ management framework (Figure 2). The user can start the execution of the request, which behind closed doors consists of the automatic invocation of the company or third party services. In case of failure, or new services being added to the system, the background process dynamically switches to alternatives at runtime in order to complete the user's request.

The mashup construction is achieved by enriching service interfaces with semantics (Section 5.1). Thanks to their semantic description, services are compared and linked to each other if they hold a corresponding interface relation (Section 5.2). That way, if a service input and output represent similar semantic concepts, the output of one of the services can be used as input for the other. On the other hand, the result of the execution of a service may correspond to a condition required for the execution of another service.

The automatic construction of a new application is triggered by the definition of a request by the application manager. Planning algorithms (Section 5.3) combine the available semantically enriched services into service mashup graphs. Each service node delivers results to the parent services calculating the defined application request. Recovery mechanisms replace failed service points with alternative service (mashups) at runtime (Section 5.4).

The following requirements are taken into account in detail during the design of the WTE+ management framework:

1. Usability - both domain experts within a business context and end-users should be able to construct and share their custom-made mashups.

2. Semantics - automating the mashup construction requires a system capable of understanding the meaning of the available data and services.

3. Quality of Service - in case of equivalent services or alternative compositions for calculating the same piece of information, a selection should be made based on the required QoS constraints (optimization of the cost to execute a given service, its execution time or both parameters).

4. Performance \& Scalability - due to the increase of the number of services, reducing the search space of available services is of crucial importance.

5. Dynamic mashup adaptation - the enormous changes in the domain of services require a dynamic system able to adapt to new requirements, services, providers, QoS parameters, and failure points.

6. Framework Modifiability - processing of a user's request consists of mashup composition, service selection based on QoS parameters and execution. Implementing the different steps as separate components enables the creation of new ones suiting diverse user requirements.

7. Transparency - in order to control the internal operations, a comprehensive monitoring feature is required for the generation of logging messages as automated systems generally lack transparency. 


\section{Related Work}

This section introduces concepts such as Service-Oriented Applications, Semantic Web, and composition algorithms and focuses on related work in the context of existing mashup composition and execution frameworks.

\subsection{Service Component Architecture and the Semantic Web}

The Service Component Architecture (SCA) (Beisiegel et al 2005) provides a set of specifications describing a model for building Service-Oriented Applications (SOAs) (Cândido et al 2009). It extends and complements prior approaches to implementing Web services building on their open standards. SCA encourages the use of business logic components offering their capabilities through service-oriented interfaces and consuming functions offered by other services. Building a SOA is divided into two major parts: the implementation of service components which provide services and consume other services and the assembly of sets of components to build business applications, through the wiring of service references to services. SCA emphasizes the decoupling of service implementation and of service assembly from the details of infrastructure capabilities and from the details of the access methods used to invoke services.

The advantage of this kind of platform is the reusability of the Web services and the possibility for construction of component based systems (Papazoglou et al 2008). The Semantic Web (Hawke et al 2011; Berners-Lee et al 2001) supports this by offering more access not only to content but also Web services. Languages like WSDL-S (Web Services Semantics) (Akkiraju et al 2005) and its successor SA-WSDL (Semantic Annotations for Web Service Description Language) (Verma and Sheth 2007) enrich the standard WSDL description and XML Schema with annotations in different semantic representation languages. More expressive languages like OWL-S (Web Ontology Language of Services) (Martin et al 2004), WSMO (Web Service Modeling Ontology) (Roman et al 2005), and SWSO (Semantic Web Services Ontology) (Battle et al 2005) provide a complete semantic description of Web services specifying inputs, outputs, pre- and post conditions (IOPEs), and non-functional properties. Their interaction model supports choreography (Brogi et al 2004) and/or P2P (peer-to-peer) orchestration for Web services.

The proposed framework in this article concentrates on the use of OWL-S instead of WSMO. OWL-S is more mature regarding the definition of the process and grounding model of Web services (Lara et al 2004). This presents an essential advantage as the different control constructs used in its process model offer the flexibility desired for the developed WTE+ management framework.

\subsection{Semantic Matching and Composition Algorithms}

One can differentiate between different models to be used for the automatic composition process. The state chart-based model in (Gamha et al 2007) ex- 
plores the concept of communities of heterogeneous services which propose similar functionalities presented by a homogeneous ontology. The composition process utilizes IOPEs matching of OWL-S descriptions. The OWL-S matching procedure also provides support for the functional decomposition of an application in a workflow. This principle is adopted in the Workflow Composition Tool described in (Gubala et al 2006) through the use of the Petri Nets Formalism (Hamadi and Benatallah 2003). Except for the automatic workflow construction based on the process of semantic service discovery and matchmaking this tool also deals with reduction operations, reuse of internal solutions, validation algorithms, annotation of unresolved dependencies, and storage of service descriptions in a registry.

Planning algorithms are employed for solving a specified goal through the discovery and composition of Web services that are able to provide the needed calculations and data. In (Sirin et al 2004) a predefined OWL-S workflow is first translated in SHOP2 syntax and then HTN planning is executed. This system does not handle concurrency since SHOP2 has no support for OWL-S's 'Split' and 'Split+Join' control constructs and an output concept. An AI planning tool based on a backward chaining algorithm is the OWLS-Xplan (Klusch et al 2005) constructing a service sequence, as opposed to a mashup graph, using an ontological definition of the initial and the requested goal state. However, before planning, the OWL-S 1.1 service descriptions are first converted to corresponding PDDL 2.1 (Planning Domain Definition Language) descriptions which could raise performance issues. The PDDL planner is in turn a linear STRIPS planner extended with HTN planning. On the other hand, The Web Service Composer suggested in (Sirin et al 2004a) plans immediately in OWL$\mathrm{S}$ but the needed composition is constructed semi-automatically requiring user feedback at each service matching step.

A Non-Backtrace Backward Chaining method is proposed by the authors of (Liu et al 2004; Zhang et al 2005) as the efficiency of a backward chaining algorithm is affected due to the possibility for backtrace especially when the number of rules is very large. Thanks to the definition of an input closure of the yet unresolved inputs, the backtrace problem is solved and the construction of a composition plan is converted in BPEL4WS (Business Process Execution Language for Web Services) for execution.

\subsection{Service-Oriented Composition and Execution Frameworks}

Several research projects some of which within the European Union Sixth and Seventh Framework Programme aim at creating platforms supporting the runtime creation, management and execution of service mashups. The offered levels of automation start from manual creation of a composite service mashup out of existing components and going as far as pro-active failure prevention and mashup adaptation. Most of these platforms require skilled web developers. 


\subsubsection{Manual Mashup Construction}

The SODIUM (Tsalgatidou et al 2006) and OPUCE (Yelmo et al 2009) projects consist of a set of languages, tools and related middleware for the creation and execution of workflows composed of heterogeneous services. Specifically, SODIUM supports abstract as well as concrete modeling of workflows (by providing a Visual Service Composition Language and Editor), uniform discovery of constituent heterogeneous services (through a Unified Service Query Language and Engine) and execution of service workflows (through the Unified Service Composition Language Engine). The OPUCE project aims to create a complete platform to support the creation, management and execution of usercentric services in telecom-oriented platforms. Here, services are envisioned as short-lived telecom services that end-users create by orchestrating simpler services using the Web and Mobile Editor Tool. The newly created services may be published and shared with other users. Within OPUCE the execution platform is considered to provide enough resources to provision all components and deploy the services.

Similar to SODIUM and OPUCE the WTE+ management framework focuses on the composition of service workflows within the enterprise environment. It provides a user interface supporting the manual revision of the resulting service mashups. The difference is the enrichment of these services with semantic descriptions in OWL-S. This approach enables the implementation of planning algorithms by the WTE+ management framework supporting the automatic instead of manual construction of new service workflows. These are constructed starting from a user-defined initial and goal state through semantic matching of service effects to required preconditions (detailed below in Section 5.3).

\subsubsection{Automated Service Composition}

Platforms such as Amigo (Valle et al 2005) and INFRAWEBS (Agre and Marinova 2007) propose approaches, in which the process of finding appropriate services is guided by algorithms for decomposition of user goals into sub-goals and discovering the existing services able to satisfy these sub-goals without further planning. Amigo develops middleware that dynamically integrates heterogeneous systems to achieve interoperability between services and devices. Home appliances, multimedia players and renderers and personal devices are connected in the home network to work in an interoperable way. Amigo uses its own extension to OWL-S: Amigo-S providing support for describing functional and non-functional properties of the Amigo middleware services and the underlying communication mechanism. INFRAWEBS achieves compatibility of services participating in the composition by using a consistent description of a composite goal template, prepared by the service application provider at design-time. Using only implicitly provided information about the desired order of execution of services in the composition, the proposed algorithm is able to find a proper orchestration of services in the composition as well as to 
discover the appropriate service substitutions when some of the services in the composition cannot be executed due to some physical reasons.

The architecture in (Fujii and Suda 2006) consists of a Component Service Model with Semantics (CoSMoS), a Component Runtime Environment (CoRE), and a Semantic Graph based Service Composition (SeGSeC). CoSMoS models components from three aspects: functional (operations and properties using data types), semantic (representation of each property of the component), logical (rules to extract the semantics from a workflow defining conditions and consequences). It defines a set of inputs, outputs, properties, operations and exceptions and uses semantic graphs to link properties to components. CoRE provides interfaces to discover and access components modelled by CoSMoS. SeGSeC composes an application by discovering components through CoRE, and synthesizing a workflow of the application based on the semantics of the components modelled by CoSMoS.

The developed planning algorithms of the WTE+ management framework construct service mashups based on previously defined user requests. In contrast to Amigo and INFRAWEBS, the user goals are not only decomposed into sub-goals matched to existing services, but further extended into a full workflow in case of missing data. Based on a semantic description of initial state information and user defined goals a service plan is generated proposing a partially ordered network of services that each contribute at describing the desired service mashup. Instead of using custom semantic templates much like CoSMoS, planning is immediately performed in OWL-S, adopting the richness of the OWL-S control constructs such as 'Split+Join', 'IfThenElse', 'ForEach', 'Choice'. Novelty with respect to the presented related work is the automatic addition of such control constructs depending on the calculated semantic dependencies between the selected services resulting in complete workflows (detailed below in Section 5.2).

\subsubsection{Service Orchestration}

While from one hand the previous platforms focus on the centralized composition of services, platforms such as SOA4All (Lecue et al 2009), mashArt (Daniel et al 2009), CRUISe (Pietschmann 2009), MarcoFlow (Daniel et al 2010), MashWeb (Pfeffer 2009), MashLight (Baresi and Guinea 2010), and Astro (Pistore et al 2005; Bertoli et al 2010) focus on the orchestration between the individual services. SOA4All offers a template-based assisted service composition shielding the end-users from the semantic links and dependencies between the assembled services. Its focus is on the manual creation of data flows controlling the output-input flows and workflows controlling the execution sequence of the services. MashArt, CRUISe and MarcoFlow facilitate dynamic, service-oriented composition of user interfaces (UIs) for modern Web applications. MashArt provides for a simple editor and a lightweight runtime environment running in the client browser. It focuses on the orchestration of distributed (i.e., multi-browser) applications and multiple actors. Complex features like transactions or exception handling are outside its scope. CRUISe re- 
sembles mashArt, especially regarding the componentization of UIs. However, there is no support for the seamless integration of UI components with service orchestration (i.e., no support for complex process logic). CRUISe rather focuses on adaptivity and context-awareness. It provides UIs "as-a-service" that can thus be selected, customized and exchanged with respect to the current context. None of them however supports the coordination of multiple different actors inside the same process, and the distribution of UIs over multiple browsers. MarcoFlow extends BPEL (BPEL4UI) to generate the UI composition logic (that runs entirely on the browser, for performance reasons) and the server-side logic that performs service orchestration and distributed UI synchronization between browsers. The platform targets skilled web developers familiar with BPEL and complex applications that possibly involve multiple actors distributed over the Web, but that need orchestration.

The MashWeb suite of Web components supports late binding and distributed orchestration of services within composed mashups. It dynamically integrates 3rd party services into new applications during runtime and distributes them between the client and server side. This distribution allows for integration of local resources residing on the users' devices and a widget-like offline capability. It creates data flows controlling the output-input flows and workflows controlling the execution sequence of the services. This approach enhanced with actual execution flows is also taken by MashLight targeting lightweight devices enabling the creation of mashups using Web 2.0 widgets by not necessarily technically experienced users. The proposed solution uses control flow templates to semi-automatically create processes based on the end user's needs.

Astro translates BPEL4WS processes into states and uses knowledge level planning algorithms for composition. Comparable to semantic languages it uses the EAGLE language for expressing requirements. Service composition is achieved through the association of inputs and outputs of services. The result is automatic generation of BPEL4WS process interacting with existing BPEL4WS processes through the extraction of interactions with the composite service (communication and transition steps). Future works aims at integrating semantic languages such as OWL-S and WSMO into the orchestration process.

In contrast to these platforms, the WTE+ management framework takes up a centralized approach of service composition. Instead of using BPEL (SOA4All, MarcoFlow, Astro), services are enriched with semantic descriptions using OWL-S. The result is that mashup construction is immediately performed in OWL-S, adopting the richness of the OWL-S control constructs which are automatically added (not supported by CRUISe) depending on the calculated semantic dependencies between the selected services. These control constructs define the data and workflow links between the selected service blocks which is similar to the definition of the service orchestration supported by MashWeb and MashLight. These links are automatically combined into a single service workflow (separated data-flow and workflow in MashWeb, control flow template in MashLight). The WTE+ execution techniques support 
the actual invocation of the individual services and the forwarding of their results to the right components (detailed below in Section 5.2).

Astro is closely related to the WTE+ approach as it implements planning algorithms for composition. Disadvantages are the necessity for translation between BPEL4WS processes and states, the use of the EAGLE language comparable to a semantic language, service composition based on inputs and outputs, not taking into account complex service preconditions and effects. In the future they plan integrating the use of actual semantic languages such as OWL-S and WSMO.

An advantage of the semantic approach is the automatic classification of services into groups of equivalent semantic interfaces. During the actual service execution monitoring data on service performance is collected. In case of service failures, WTE+ is able to automatically switch between equivalent semantic services or construct a partial mashup resolving the failed point (as opposed to MashArt) resuming the execution (detailed below in Section 5.1.2).

\subsubsection{Automated Service Selection and Composition Reconstruction}

The composition and service orchestration process of these approaches require low level QoS-based service selection. The workflow engine WSQoSX (Web Services Quality of Service Architectural Extension) in (Berbner et al 2006) uses heuristics for late binding of services at runtime and dedicated accounting and monitoring of QoS. Goal is maximizing the overall QoS of the composition by taking into account user preferences and monitoring SLA violations at runtime. The approach assumes sequential Web service compositions. (Zisman et al 2008) concentrates on dynamic service discovery based on characteristics of the services: structural, behavioural, quality and contextual. It supports service pull search during application deployment and push search during application execution in case of required alternative services. Service matching is based on the computation of distances between query and service, matching of names and parameter data types, semantic categories, behavioural state transitions. This requires the definition of alternatives used for adaptation during the execution through the use of pre-defined replacement policies to switch services. PAWS (Process with Adaptive Web Services) (Ardagna et al 2007) supports process design and execution using a BPEL engine. It selects the best available services for executing the process and defines the most appropriate global and local QoS constraints for delivering them. PAWS offers design-time specification of all information required for the automatic runtime adaptation according to dynamically changing user preferences and context. Services are selected using a similarity-evaluation algorithm for naming and structural comparisons of the WSDL interface and semantics through WordNet and SAWSDL. Monitoring and repair capabilities tackle service provisioning in case of failures through recovery and self-adaptation. (Baresi et al 2007) covers deployment of adaptable Web service compositions. It consists of a distributed registry (DIRE), service composition execution (SCENE), and dynamic monitoring (DYNAMO). DIRE connects a set of heterogeneous reg- 
istries. The SCENE language represents a definition of service compositions extending BPEL with rules (Drools) for self-reconfiguration (designer defines rules for runtime adaptability) that disciplines the way the composition evolves at runtime by applying proper binding and re-configuration policies. Service recovery is also handled in (Lin et al 2010) where faulty services and neighbouring ones are replaced in order to maintain defined QoS using BPELQ (structural flow + QoS). Services are classified based on functionality with different QoS. Function replacements are 1:1, 1:many (expansion of the configuration region in order to find suitable replacements), many:1 provided by a mapping database beforehand by service experts.

MoSCA (Prete and Capra 2008) facilitates the rapid development and deployment of reliable composite services. At design-time, a MoSCA Service is uniquely identified within an OWL-S ontology, and described as a composition of further MoSCA Services, which can themselves be composite or basic. At run-time, whenever a (composite) service is invoked, MoSCA selects the providers, among those currently available that are capable of collectively delivering the (composite) service with the highest reliability. Reliability is estimated by reasoning about providers' historical collocation patterns that are learned over time. Unforeseen changes to such patterns are being monitored as well, potentially triggering re-bindings during service execution.

(Tosi et al 2009) focus on autonomous reaction to change in implementation of the services, detecting integration mismatches and triggering dynamic execution of suitable adaptation strategies. It uses a taxonomy of integration faults in order to anticipate potential mismatches as checklist for mismatches and test cases, consisting of 9 categories. In case of a mismatch, an update of the structure and behaviour of the client application is triggered. The platform requires a software architect to analyze and define mismatches, test cases and adaptation strategies.

An advantage of the semantic approach of WTE+ is the automatic classification of services into groups of equivalent semantic interfaces each having different QoS parameters. This allows for late binding in order to select the services offering the desired QoS for execution. MoSCA and (Lin et al 2010) propose a similar classification based on respectively semantics and functionality. During service execution monitoring data on service performance is collected, updating the QoS parameters resulting in adaptation of future executions. In addition, in case of failures, the planning algorithms automatically replace the failed services by equivalent ones or construct partial service mashups. This eliminates the need for manual design-time specification of alternatives as required by (Zisman et al 2008), PAWS and (Tosi et al 2009). Similar to PAWS and SCENE the standard compositions are tailored to the personal needs of the users through the definition of business logic rules enabling the automatic personalization of his requests. Both solutions adapt BPEL instead of the more flexible semantic approach of WTE + that is able to respond to unplanned conditions (detailed below in Section 5.4). 


\subsubsection{Pro-active Monitoring and Failure Prevention}

Last enhancement covers the pro-active QoS monitoring and failure prevention. Several different techniques are adopted. (Miyagi et al 2004) generates probability function for the response time of an application over the TCP/IP layer. In (Canfora et al 2005) runtime replanning is supported through prediction if the actual QoS will deviate from the required SLA. New information on QoS going beyond estimated percentage of SLA violations triggers replanning of a slice. The approach uses a proxy service for semantic service discovery and binding in order to optimize the fitness function of the composition. (Dai et al 2009) also maximizes the fitness function of the composition QoS. It predicts the performance (data transmission speed based on semi-Markov model) in order to be more effective when meeting the QoS requirements during selfhealing, monitoring and quantifying the reliability of the services. Result is the automatic switch to replacements before execution of the failed component. (Metzger et al 2010) uses online testing producing failure predictions with specific confidence level resulting in pro-active adaptation if high enough certainty that the failure will occur. (Guinea et al 2011) focuses on multi-layered control loops monitoring and adapting BPEL processes deployed onto a dynamic infrastructure. Infrastructure and software events are monitored based on data from running applications in order to detect anomalies. Following is event correlation aggregating these data from multiple sources producing data at a higher level of abstraction using machine learning to find relations between set of metrics and the key performance indicators. Adaptation actions are manually defined by the user in the BPEL process. Machine learning is also adopted by PREvent (Leitner et al 2010) for event-based monitoring and prediction of SLA violations and automated runtime prevention by triggering adaptation actions in service compositions. It uses a registry for Web service metadata and QoS monitoring info. For each composition metric it registers event listeners. Adaptation actions should be predefined and chosen at runtime depending on the required prevention steps. It provides support of data flow manipulations, 1:1 service rebinding, add/remove activities at runtime but there is no support for structural adaptations and it does not take the cost of adaptations into account. ProAdapt (Aschoff and Zisman 2011) (Proactive adaptation of service composition) is based on the exponentially weighted moving average (EWMA) for modelling service operation response time. Decision for replacement not only takes into account this response time but the cost value as well. It consists of a monitor verifying the QoS aspects of the execution and adapter using these data for adaptation of the composition.

The WTE+ management framework does not support pro-active monitoring and prediction of QoS parameters. On the other hand, while most of the applications require predefined adaptation and prevention actions, WTE+ monitors and reacts at runtime by automatically planning recovery actions. This mechanism is enhanced with the possibility for several (partial) iterations of mashup adaptation and execution. Constructed mashups are partially executed and the intermediary results are used as feedback to further tune the 
service mashup at runtime (detailed below in Section 5.4.2).

In conclusion the WTE+ management framework adopts several of the mentioned above mashup creation and execution techniques enhancing them with automated use of control constructs and dynamic adaptation to changing runtime environment. The proposed algorithms offer several levels of automation starting from manual selection of matching services to required interfaces, to completely automatic data and workflow construction, QoS-based service selection, service orchestration during mashup execution and runtime failure recovery. The framework is designed in a way that different QoS-aware planning algorithms and execution engines are easily plugged in just by extending the respective interfaces.

\section{Framework Design Details}

This section focuses on the architectural design of the WTE+ management framework enabling dynamic development of service mashups. The implementation specific details of the main functional components follow in Section 5.

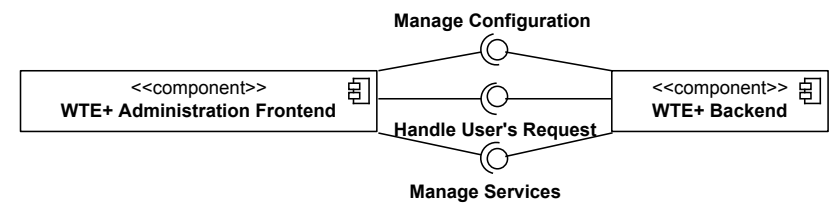

Fig. 3 High level architecture of the WTE+ management framework consisting of the frontend configuration interface and backend dynamic mashup construction.

The WTE+ management framework is split up into an Administration Frontend and Backend as detailed in Figure 3. The Frontend, implemented as a JavaEE Web application, represents the user interface for the application manager which main functionality consists of the configuration of the available services and their QoS parameters, the definition of users' requests needed for the construction of new service mashups, monitoring of the backend processes, and the visualization and possible manual adaptation of the constructed mashup graphs. The Backend exposes the service repository, the service matching strategies, and the dynamic construction and execution of the service mashups.

\subsection{WTE+ Frontend}

The Frontend in Figure 4 is a portal to the actual mashup construction framework. Its Request Management Module is the main interface for processing users' requests. It consists of 4 parts in Figure 5 and 1 popup Frontend Applet in Figure 6. 


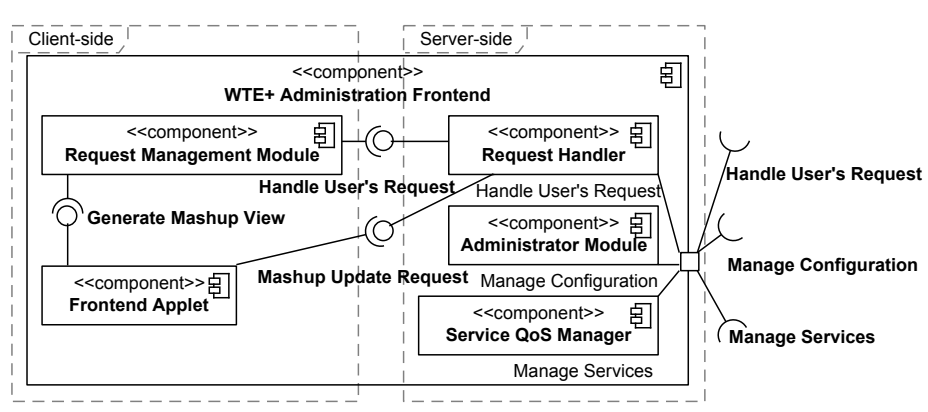

Fig. 4 The WTE+ Frontend is a user interface for the definition of users' requests and the configuration of the framework.

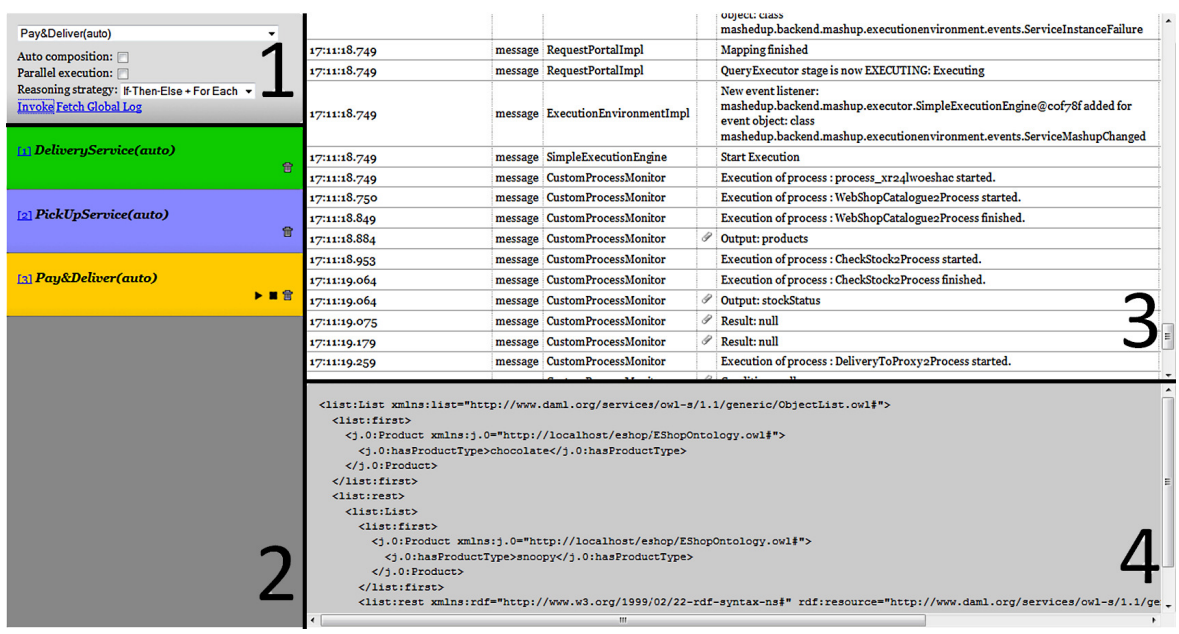

Fig. 5 The Request Management Module is responsible for the definition and monitoring of users' requests.

- Part 1 provides an overview of the available services. The user can upload a new request and configure several parameters such as the type of mashup processing (divided into 3 stages: mashup composition, service mapping and execution or all the stages immediately performed in one go), parallel or serial execution of independent service paths in the mashup graph, reasoning strategy (basic without control flows, with control flows or complete composition expanding the alternative paths).

- Part 2 displays the status of the ongoing requests using colour codes depending on their stage: yellow for a request in execution, green for a finished request and red for a failed request.

- By selecting a specific request monitoring information is tracked in part 3 where timestamp, executing module, monitoring message and state are found. 


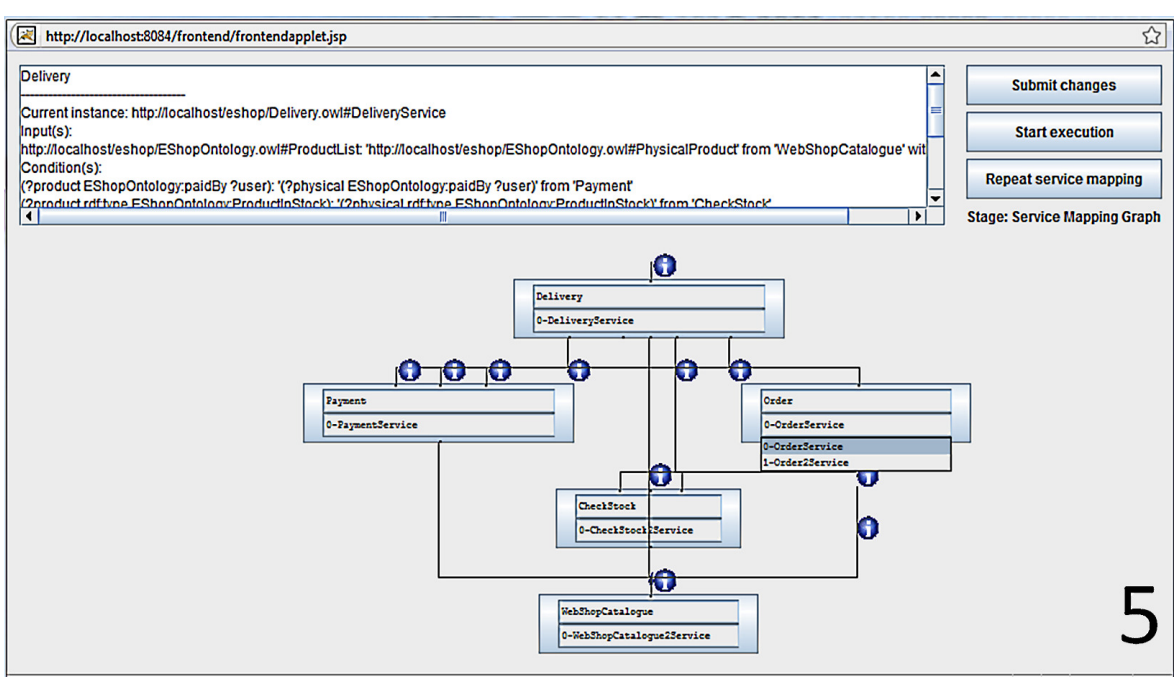

Fig. 6 The Frontend Applet visualizes the constructed mashup graph and selected services for execution.

- Messages containing paper clips as links to more data are loaded in part 4. These are amongst others XML representations of a goal request, semantic composition graph, mapping graph, and execution results.

- The composition and mapping graphs are visualized in part 5, the popup Frontend Applet. There the user is able to manually tune the composed mashup and selected service instances to his specific needs and start new compositions and executions.

The Request Handler, constructed as a servlet, receives HTTP requests from the Request Management Module, interprets them and calls the corresponding methods in the Backend. Next to framework configuration (e.g. resetting, initializing and shutting down the Backend) the Administration Module provides functionality for the addition and removal of services. In order to register a service it is sufficient to indicate the URI of its OWL-S description. Similar to this the user can also register new services using the Service QoS Manager where he is able to indicate various properties such as statefull or stateless, QoS parameters (e.g. execution time and financial cost), status and cache timeout. The currently active Web services are showed in groups of equivalent semantic interfaces classified by the Backend.

This architecture supports the transparency requirement (req. 7) defined in Section 2 enabling a developer with the overall management and monitoring of the automated mashup composition process.

\subsection{WTE+ Backend}

The main building blocks of the Backend are presented in Figure 7. Requests are processed by the Coordinator which handles the communication 


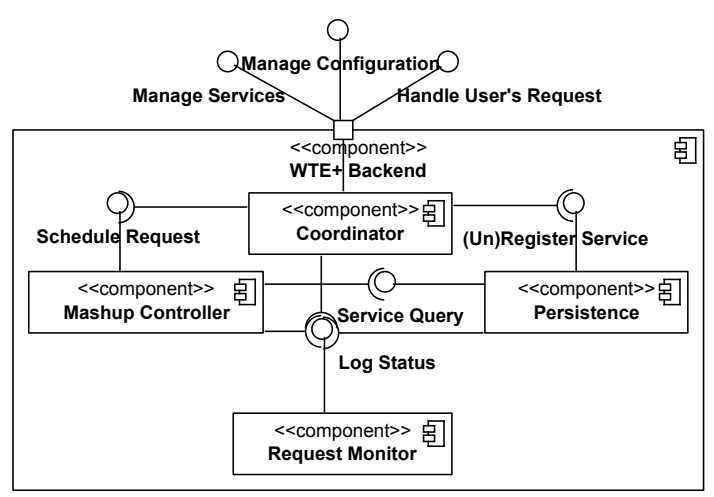

Fig. 7 The WTE+ Backend manages the service repository, monitoring module and the dynamic construction and execution of service mashups.

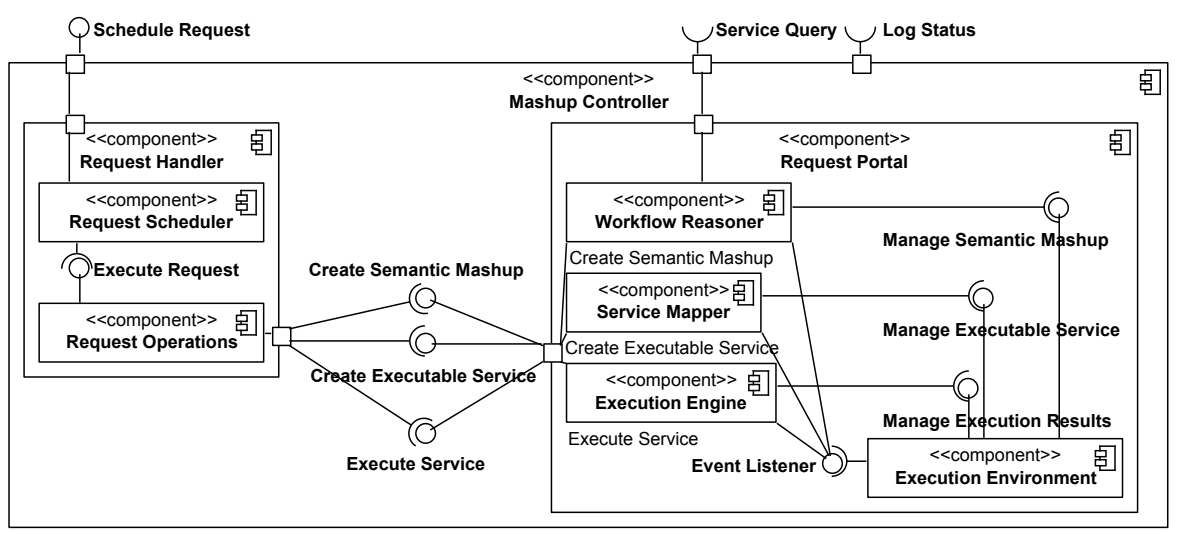

Fig. 8 The WTE+ MashupController consists of planning algorithms for the construction and execution of service mashups.

with the mashup monitoring module (Request Monitor), the service repository (Persistence), and the mashup creation and execution environment (Mashup Controller). The Request Monitor provides logging information, constructs XML representations of the service mashup, the utilized resources for execution, and intermediary results which in turn are presented by the Frontend. The Persistence component manages the available services, their QoS parameters, groups equivalent semantic descriptions (Section 5.1) and enables querying for required matching service interfaces during mashup construction (Section 5.2).

The Mashup Controller in Figure 8 handles the actual construction and execution of service mashups (Section 5.3). The Request Scheduler provides a management interface keeping track of scheduled and executing user's requests. Depending on the user preferences configured in the Frontend different Request Operations are selected handling a single user's request. These Operations represent different Workflow Reasoners, Service Mappers 


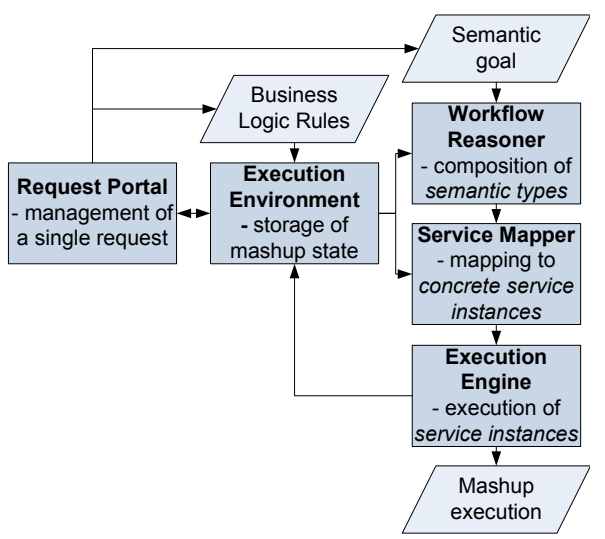

Fig. 9 Process view of the mashup creation, execution and runtime adaptation for a user's request.

and Execution Engines or combinations of the three resulting in a plug-in architecture.

The mashup composition and execution process is illustrated in Figure 9. A more detailed description of the following main components follows in Section 5 .

1. Starting from a semantic definition of a user-defined request, the Workflow Reasoner constructs a service mashup using the available semantically enriched services.

2. The Service Mapper converts this mashup into an executable process by selecting the specific service instances for each building block of the mashup (Avellino et al 2008) satisfying the predefined QoS constraints and requirements (execution time, cost) and defining the necessary bindings between them.

3. The process is executed by the Execution Engine handling the invocation workflow of the service instances through the use of the OWL-S API (Möller 2010). This API provides programmatic access to create, read, write, and execute OWL-S described atomic and composite services with WSDL or UPnP groundings. In combination with the WTE+ management framework it supports the Execution Engine through the invocation of service mashups utilizing control constructs such as 'Sequence', 'Split+Join', 'IfThenElse', 'ForEach', 'Choice'.

4. The Execution Environment acts as a storage for users' requests, business logic rules (e.g. desired service instances), inputs, results, and execution state of the service mashup.

5. The Reasoner and Mapper use these intermediary results to optimize the reasoning and mapping process of the service mashup at design and runtime (Section 5.4). 


\section{Framework Implementation Details}

This section focuses on the novel contributions of the WTE+ management framework starting with a discussion on the grouping of equivalent semantic services. Following is an overview of the matching methods between these components during the mashup construction process including automated addition of control constructs. Furthermore, the planning algorithms for the actual composition process are detailed together with the at-runtime reconfiguration and adaptation.

\subsection{Management of Service Instances}

The following sections describe the use of the quality of service parameters by the WTE+ management framework, the semantic enrichment of service interfaces and the definition of user's requests.

\subsubsection{Service Instance QoS}

A distinction is made between two types of components used by the presented framework: concrete service instances and abstract semantic types.

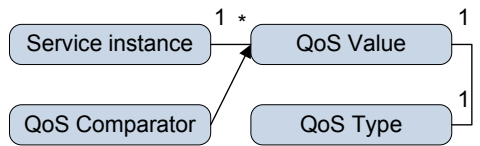

Fig. 10 Definition of the Quality of Service linked to a specific service instance.

Concrete services instances are the actual services executed on specific resources. Each service instance is provided with several QoS parameters describing its properties. Examples include the average execution time, financial parameters, and availability. They are defined beforehand or their values are dynamically adjusted by the Execution Engine based on previous invocations. For example, the average execution time is updated after each invocation of a service. The QoS of a specific service instance consists of a QoS Type which can amongst others be the economic cost for executing a service or the execution time. Each QoS Type has a QoS Value and specific QoS Comparator comparing the actual QoS Values (Figure 10). These QoS Types are assigned different priorities by defining a weight factor to the QoS Value at design or runtime. After the composition phase the Service Mapper will select a specific service instance for each semantic node of the constructed graph based on the comparison of the weighted QoS Values calculated by the QoS Comparator. The result is different service selection for the same mashup graph depending on the updated service QoS Values. This offers an application manager with the possibility to extend the framework with new QoS parameters, define 
customary comparison techniques and specify different priorities of the QoS parameters favouring one or more of them.

\subsubsection{Semantic Description of Service Instances}

In the presented framework, these service instances are enriched with semantic annotations using OWL-S. Instead of defining their inputs and outputs using XML Schema types much like WSDL they are expressed by ontological concepts in OWL. For the definition of the service preconditions and effects SWRL (Semantic Web Rule Language) (Horrocks et al 2004) expressions and built-ins (SWRLB) such as comparisons are adopted. An SWRL expression consists of a property and one or more arguments (semantic OWL concepts). Examples include an OWLClass with one argument ${ }^{1}$, an ObjectProperty with two arguments ${ }^{2}$, a DataProperty with one argument and an RDF type ${ }^{3}$, a SWRLB primitive with one or more arguments ${ }^{4}$.

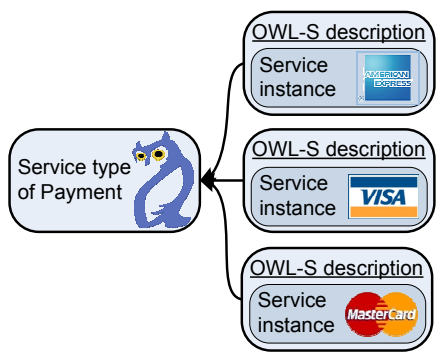

Fig. 11 Grouping of semantically equivalent payment services into one semantic Payment type.

As several semantically equivalent service instances (equivalent IOPEs) exist, their semantic interfaces are grouped into a single semantic type, thus reducing the search space of available instances. For instance, Figure 11 details the grouping of several payment methods (bank transfer, Visa, PayPal) into one semantic payment type. The OWLS-MX Matchmaker (Klusch et al 2009) provides a partial solution to this classification problem by comparing service inputs and outputs and assigning a score based on the semantic distance between these concepts. As a truly equivalent match between two semantic interfaces cannot be expected, the services are grouped in a hierarchical fashion. Although exhaustive enough, the OWLS-MX solution lacks the ability to compare service preconditions and effects. Therefore, its functionality is extended with support of comparison between concept properties, equivalent

\footnotetext{
1 InStock(Product)

2 paidFor(Customer, Product)

3 hasName(Customer, String)

4 equal(StockStatus,true)
} 
SWRL expressions and matching build-ins. This enables the Workflow Reasoner to search for a specific group of services producing required outputs and more importantly effects during the composition of the service mashup. Afterwards the Service Mapper will select a corresponding service instance offering required QoS.

\subsubsection{Definition of a User's Request}

The semantic types not only refer to concrete service instances but also represent users' requests and newly created mashups. In order to specify a request the user has to instantiate concepts from predefined OWL ontologies using an editor such as Protégé (Stanford 2011). This editor provides support for OWL, RDF (Resource Description Framework) and XML Schema making it possible to easily design ontologies through a graphical interface. The user's request consists of two definitions: (i) initial state defined by inputs (instantiated OWL concepts) and valid conditions (properties between these concepts) and (ii) user-goals defined by required outputs (instantiated OWL concepts) and effects (properties between these concepts). On one hand the initial state is information that holds true at the moment of the request and can be used as input data. The example below shows the definition of a specific product chocolate and an authenticated user.

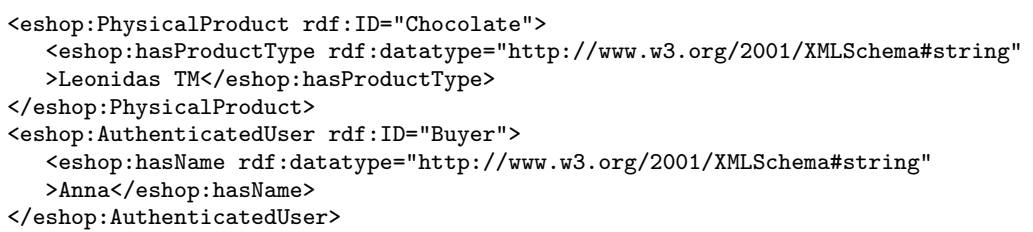

On the other hand, a goal state describes the required end state of the composed application after execution. In the example an authenticated buyer should have paid for the specific product and this product should be delivered to the person in question.

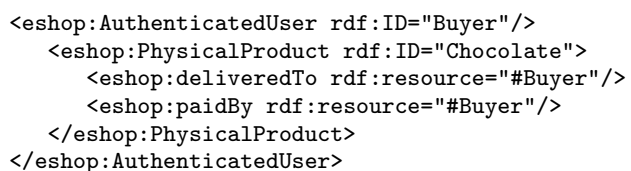

These initial and goal states $(\mathrm{RQ}=\mathrm{IS}+\mathrm{GS})$ are transformed by the WTE+ management framework into provided inputs and valid preconditions ( $\mathrm{IS}=\mathrm{I}+\mathrm{P})$ and required outputs and effects $(\mathrm{GS}=0+\mathrm{E})$ resulting in an abstract semantic service description. In the e-shop example this definition states that the abstract service requires an authenticated user and a product as inputs and its execution will result into payment and delivery of this product to the authenticated user. The following examples present the specific transformation of the initial and goal state:

- Service inputs from the initial state defining a Buyer as an authenticated user type and a physical product Chocolate: 
<process: Input rdf:ID="Buyer">

$\langle$ rdfs: label>buyer</rdfs: label>

<process:parameterType rdf:datatype="http://www.w3.org/2001/XMLSchema\#anyURI"

$>$ http://localhost/eshop/EShopOntology.owl\#AuthenticatedUser</process: parameterType > $</$ process: Input $\rangle$

<process: Input rdf:ID="Chocolate">

<dfs: label>chocolate</rdfs: label>

<process:parameterType rdf:datatype="http://www.w3.org/2001/XMLSchema\#anyURI"

$>$ http://localhost/eshop/EShopOntology.owl\#PhysicalProduct</process : parameterType> $</$ process: Input $>$

- Service preconditions from the initial state specifying 'Anna' as the buyer's name and 'Leonidas TM' as the chocolate brand:

<process: hasPrecondition>

<expr:SWRL-Condition rdf: ID="InitialStateCondition">

〈swrl: DatavaluedPropertyAtom>

<swrl:propertyPredicate

rdf : resource="http://localhost/eshop/EShopOntology.owl\#hasProductType"/>

<swrl:argument1 rdf:resource="\#Chocolate"/>

<swrl:argument2 rdf:datatype="http://www.w3.org/2001/XMLSchema\#string"

$>$ Leonidas $\mathrm{TM}</$ swrl:argument2>

$</$ swrl:DatavaluedPropertyAtom $>$

<swrl: DatavaluedPropertyAtom>

<swrl:propertyPredicate

rdf:resource="http://localhost/eshop/EShopOntology.owl\#hasName" />

<swrl:argument1 rdf:resource="\#Buyer"/>

<swrl:argument2 rdf:datatype="http://www.w3.org/2001/XMLSchema\#string"

$>$ Anna</swrl:argument2>

$</$ swrl: DatavaluedPropertyAtom>

$</$ expr:SWRL-Condition>

$</$ process: hasPrecondition $>$

- Service outputs from the goal state defining a Buyer as an authenticated user type and a physical product Chocolate. An optimization of the creation process is dismissing outputs that are already defined as inputs: <process: Output $\mathrm{rdf}: \mathrm{ID}=$ "Buyer" $>$

$<$ rdfs:label>buyer</rdfs:label>

<process: parameterType rdf:datatype="http://www.w3.org/2001/XMLSchema\#anyURI"

$>$ http://localhost/eshop/EShopOntology.owl\#AuthenticatedUser</process: parameterType> $</$ process:Output $>$

<process:Output rdf:ID="Chocolate" $>$

$\langle$ rdfs: label>chocolate</rdfs: label>

<process : parameterType rdf:datatype="http://www.w3.org/2001/XMLSchema\#anyURI"

$>$ http://localhost/eshop/EShopOntology.owl\#PhysicalProduct</process : parameterType> $</$ process:Output $>$

- Service results from the goal state defining the payment and delivery of the chocolate brand 'Leonidas TM' to the buyer 'Anna':

$\langle$ process: hasResult>

<process: Result rdf:ID="Result">

<process: hasEffect>

<expr:SWRL-Expression rdf:ID="DeliveryPayment">

<swrl: IndividualPropertyAtom>

<swrl:propertyPredicate

rdf : resource="http: //localhost/eshop/EShopOntology. owl\#paidBy" />

<swrl:argument1 rdf:resource="\#Chocolate"/>

<swrl:argument2 rdf:resource="\#Buyer"/>

$</$ swrl: IndividualPropertyAtom $>$

<swrl: IndividualPropertyAtom>

<swrl:propertyPredicate

rdf : resource="http://localhost/eshop/EShopOntology.owl\#deliveredTo"/>

<swrl:argument1 rdf:resource="\#Chocolate"/>

<swrl:argument2 rdf:resource="\#Buyer"/> 


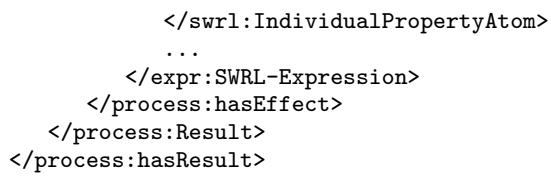

Based on this abstract service, a service mashup is composed by the Workflow Reasoner out of other service types taking the initial state definition of an authenticated buyer and selected product as input while reaching the specified goal of product payment and delivery (see Section 5.3 for more details on the composition algorithm). Its semantic description is used in turn as building block for other compositions.

\subsection{Semantic Match between Semantic Types}

Semantic types are compared and linked by the Workflow Reasoner in case of matching input-output (Hristoskova et al 2010) and/or precondition-effect relations. Depending on the quality of the match between their interfaces, control constructs are required for the construction and more importantly execution of more complex service mashups. This section gives a special attention to the use of 'IfThenElse' and 'ForEach' control constructs of OWL-S.

\subsubsection{Parametric Match}

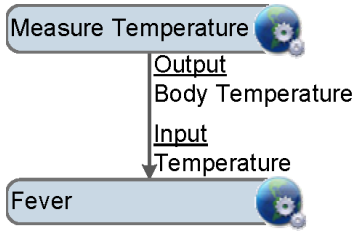

(a) Input-Output match

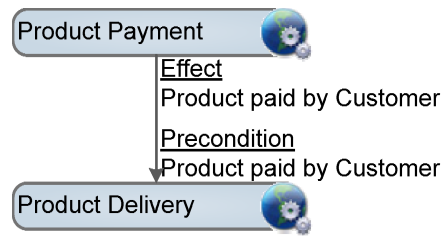

(b) Precondition-Effect match

Fig. 12 Semantic match between two service interfaces.

An input-output match between services is defined when an input and an output represent similar semantic concepts and as a result the output of one of the services is used as input for the other. As demonstrated in Figure 12(a), the output 'Body Temperature' is interpreted as a kind of 'Temperature', matching the measuring service to the service determining the patient's fever. For example, if the initial state from Section 5.1.3 did not define a specific physical product chocolate, a service will be required such as a web shop catalogue enabling the authenticated user to select products for payment and delivery. We define different qualities of semantic matches between service inputs and outputs, some of which require additional control constructs: 
- Exact. The service output exactly matches the semantic concept of the service input. S:WebShopCatalogue (0:Product) $\rightarrow$ S:Delivery (I:Product)

- Subsume. The output concept inherits from the input. This is a valid but lower quality match. S:WebShopCatalogue (0:PhysicalProduct) $\rightarrow$ S:Delivery (I:Product)

- Relaxed. The service input is more specific than the output concept in which case this is not a valid match. It is nevertheless incorporated in the composition process as a generic output can turn out to be a more specific individual after service execution. For instance, a shopping basket may consist of digital and/or physical products in which case some products need to be downloaded and others delivered. This is resolved through the use of a repository describing product individuals and their inheritance graph. In this case, the Workflow Reasoner adds an 'IfThenElse' construct between the matching services with an 'If'-condition on the specific product type output. S:WebShopCatalogue (0:Product) $\rightarrow$ S:Delivery (I:PhysicalProduct)

- List. The service output is a list of concepts matching the single input concept. For example, a shopping basket used as input for a service checking the stock status of each individual product. Here, a 'ForEach' construct is added iterating over the list of products. The specific match for each concept from the list can be all of the above (exact, subsume, relaxed). $\mathrm{S}:$ WebShopCatalogue ( $0:$ ProductList) $\rightarrow$ S:Delivery (I:PhysicalProduct)

\subsubsection{Condition Match}

A precondition-effect match is defined when the result of the execution of a service corresponds to a condition required for the execution of another service. The effect in Figure 12(b) of the product payment service realizes the payment condition for the delivery of the product to the customer. For example the user request from Section 5.1.3 requires services with results product paid by authenticated user and product delivered to authenticated user. Similar to the input-output match, different qualities of semantic matches between service preconditions and effects are defined together with the necessary control constructs:

- Exact. The service effect matches the SWRL expression representing the service precondition. A matching SWRL expression consists of an equivalent property and exactly matching semantic concepts (property arguments). S:CheckStock (E:Product InStock) -> S:Delivery (P:Product InStock)

- Subsume. The effect property still matches the precondition property and between the semantic concepts a subsume match is defined. For an ObjectProperty only the first argument can be a subtype, however if the property is defined as an inverse property, the second is also a subtype as OWL concepts inherit the properties of the super class.

$\mathrm{S}$ :CheckStock(E:PhysicalProduct InStock) $\rightarrow$ S:Delivery(P:Product InStock)

- Relaxed. This is similar to the subsume match, however one still needs to check if the concepts can be subclassed after execution as is the case in the relaxed input-output match.

$\mathrm{S}:$ CheckStock(E:Product InStock) $\rightarrow \mathrm{S}:$ Delivery (P:PhysicalProduct InStock) 
- Conditional. It should be noted that a service effect can be conditional; meaning that depending on the service output a different effect is possible. For example a service checking the stock of a product can have as effect that the product is in stock if the stock status is true or not in stock if false in which case an ordering service is added to the composition before the actual delivery of the product. In this case an 'IfThenElse' construct is added with the conditional output on the effect as 'If' statement. The following is an example of the conditional effect of the 'Check Stock' service where the product is considered in stock if the stock status is true:

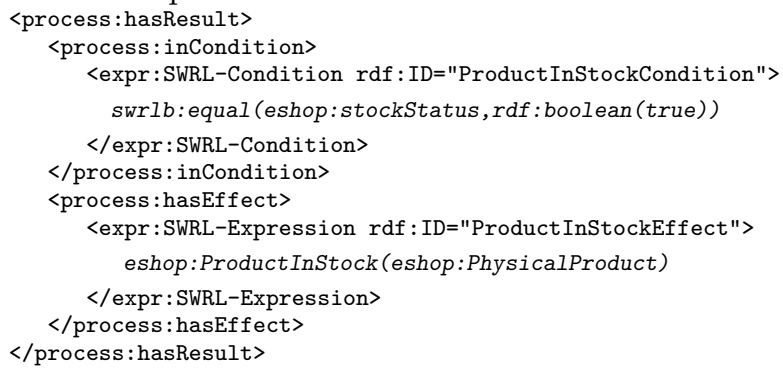

These matching strategies are used by the planning algorithms of the Workflow Reasoner during the construction of the service mashup where services are selected providing outputs and/or effects matching the required service inputs and/or preconditions.

\subsection{Automatic Mashup Creation based on HTN Planning}

A Hierarchical Task Network (HTN) plan (Sirin et al 2004; Hristoskova et al 2009 ) is a partially ordered graph of service nodes. Each service defines a certain state (i.e. the outputs and effects of the service execution) and the description of the overall state is distributed in the graph. Services of unordered nodes (in parallel paths) are executed simultaneously, through the use of the 'Split+Join' construct of OWL-S. The Workflow Reasoner in this paper adopts the HTN planning methods by incorporating the semantic grouping and matching strategies (including the use of control constructs) mentioned above. It is enhanced with runtime mashup adaptation using collected data in the Execution Environment (Section 5.4).

Planning proceeds as follows: the abstract semantic service representation of a user's request (Section 5.1.3) goes through an expansion phase followed by the actual construction through semantic matching of services (Section 5.2). The expansion Algorithm 1 queries if there are semantic service(s) mashups that already match the entire description. If so, the composition ends here. If not, it starts looking for services that are able to provide part of the required service outputs and effects (goal state (GS)). This results in splitting the abstract service in the outputs and effects $(0+E=G S)$ that need to be resolved and the inputs and preconditions $(\mathrm{I}+\mathrm{P}=\mathrm{IS})$ that can be utilized for this purpose (e.g. the payment service expects a physical product as input). Once 

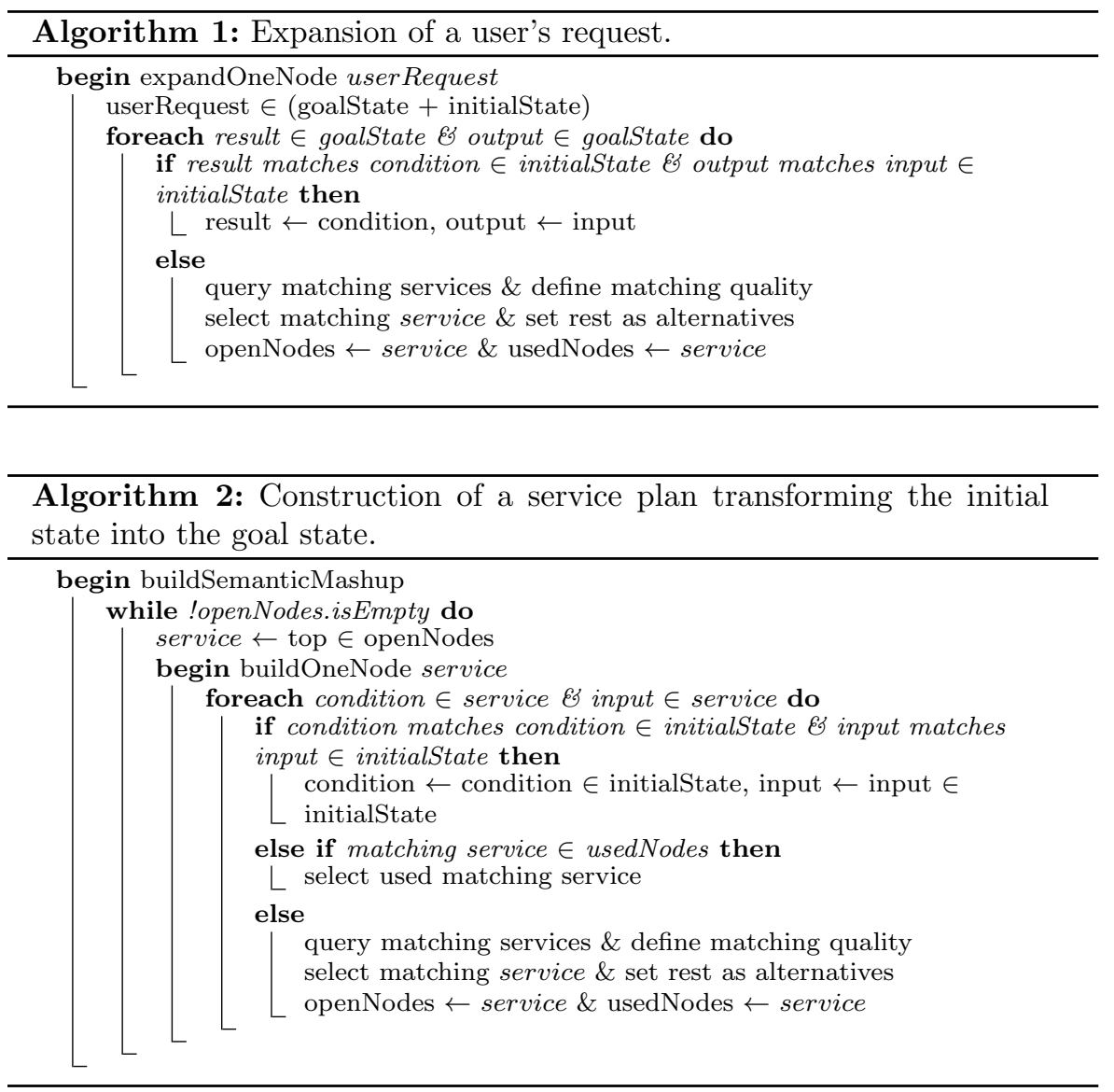

all the service outputs and effects are covered by available services, the construction phase (Algorithm 2) starts by generating a plan of services, through backward chaining, transforming the goal state into the initial state (GS->IS). This is accomplished by looping through the previously selected services and inspecting if there are additional conditions or inputs required and if the case querying for matching services. The overall process consists of queuing of found services in a breadth-first fashion constructing the shortest graph structure. The service repository is queried for matching (Section 5.2) service outputs and effects to the required inputs and preconditions of the service on top of the queue $(\mathrm{O}+\mathrm{E}->\mathrm{I}+\mathrm{P}==\mathrm{GS}->\mathrm{IS})$. Depending on the quality of the match control constructs such as 'IfThenElse' and 'ForEach' are added between the defined data links between these services. If the user selected parallel execution 'Split+Join' constructs are added between independent child graphs of a parent node. 'Choice' constructs define decision points in the execution for alternative calculation possibilities. In case no matching services are found, an exhaustive composition is presented and the incomplete inputs/preconditions 
are marked. These are provided by the application manager and/or new services are deployed filling in the missing gaps. Finally, alternatives are cached used during failure recovery.

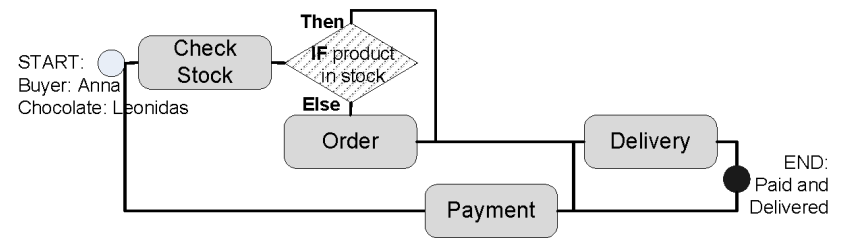

Fig. 13 Constructed service mashup graph out of the user's request payment and delivery of a physical product 'Leonidas TM' to an authenticated user 'Anna'.

The following example clarifies the HTN planning process for the defined user's request in Section 5.1.3. It is simplified by removing the input-output matching part and immediately filling in the output physical product 'Leonidas TM' and the authenticated user 'Anna'. The resulting service mashup graph is visualized in Figure 13.

I) Goal effect deliveredTo(Leonidas TM,Anna)

a) Match 'Delivery' service effect deliveredTo(physical product, authenticated user)

1) 'Delivery' condition InStock(Leonidas TM)

i) Match 'Check Stock' service with conditional effect InStock (physical product) if output stock status is true (Section 5.2)

$\rightarrow$ Set IF InStock(Leonidas TM) flow control based on 'Check Stock' output stock status

ii) Match 'Order' service effect InStock(physical product)

A) 'Order' condition notInStock(Leonidas TM)

1) Match 'Check Stock' service with conditional effect NotInStock (physical product) if output stock status is false

$\rightarrow$ Set IF NotInStock(Leonidas TM) flow control based on 'Check Stock' output stock status

b) 'Delivery' condition paidBy(Leonidas TM,Anna)

1) Match 'Payment' service effect paidBy(product,authenticated user)

II) Goal effect paidBy(Leonidas TM,Anna)

a) Match 'Payment' service effect paidBy(product,authenticated user)

\subsection{Dynamic Mashup Adaptation}

An important aspect of the presented framework is the runtime behaviour anticipating changes (e.g. availability of new services, resource and service failures) and adapting each request to user-specific requirements (business logic rules). Figure 14 presents the feedback principle if the Execution Environment 


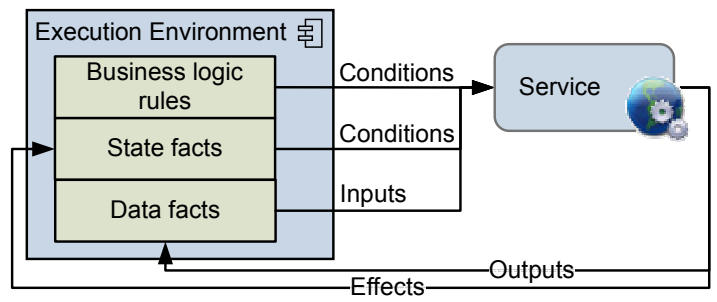

Fig. 14 Inference through matching of service preconditions and inputs and returning service effects and outputs.

where services are executed using known inputs (data facts) and conditions (state facts) and new service effects and outputs are produced and added to this environment. This results in a dynamic mashup management framework where new knowledge is inferred at runtime.

\subsubsection{Personalization of a User's Request}

Personalization of user requests is resolved in two ways. On one hand the Request Scheduler from Section 4.2 is not only responsible for scheduling of new requests but also manages finished or still in execution requests. Whenever a new user request is issued, it compares the transformed abstract service description to existing ones much like the semantic grouping described in Section 5.1. The existing request is not required to be an exact equivalent to the new one as long as its set of service effects is an equivalent subset match to the new request. The higher the number of matching service effects the similar the user requirements. A new Execution Environment is created and initialized with data (semantic types and service instances) from the matching Execution Environment. This procedure favors repeatedly selected components by users. For example if most users prefer proxy delivery then the Workflow Reasoner will be faster at constructing the e-shop mashup in Figure 15 instead of Figure 13, even though it presents a smaller graph, as it will already have an initial pool of selected matching services.

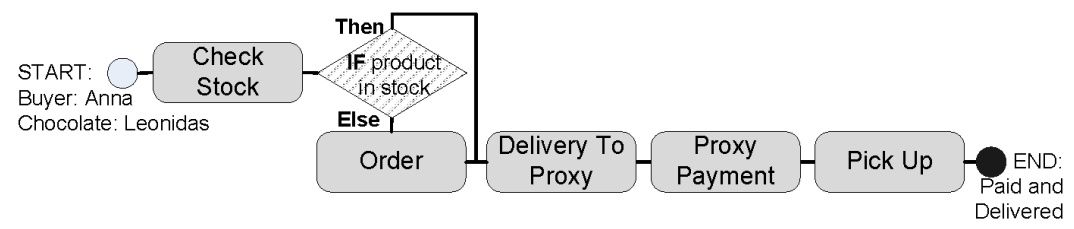

Fig. 15 Frequently selected service nodes result in the automatic construction of alternative e-shop graph that is not necessary the smallest solution.

On the other hand user-specific requests or business logic rules can be added offline or at runtime to the Execution Environment. These are used 


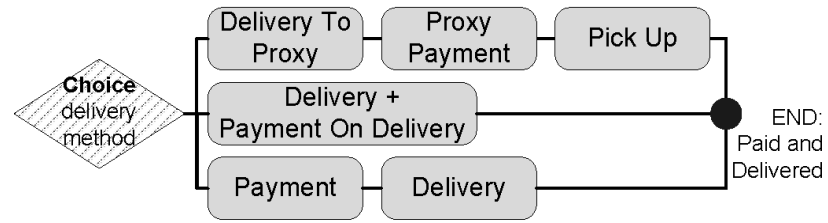

Fig. 16 Definition of a decision point between several alternative execution paths of the e-shop mashup through the automatic addition of a 'Choice' construct.

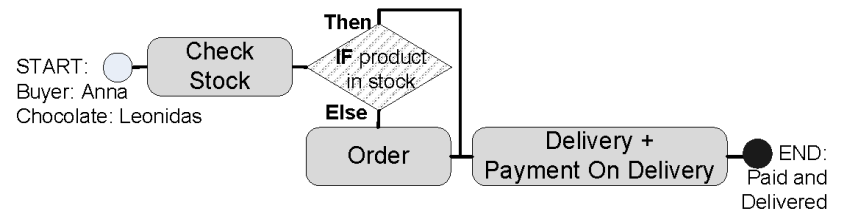

Fig. 17 Selection of a specific execution path by the user results in the automatic adaptation of the workflow by the Workflow Reasoner through the removal of alternatives.

by the Workflow Reasoner and Service Mapper to adapt the generic mashup from Section 5.3 to the user's needs at design and runtime. For example, there are several possibilities for product delivery services, visualized in Figure 16, which we define as a decision point ('Choice') in the execution: a 'Delivery' service, 'DeliveryWithPaymentOnDelivery' service, 'DeliveryToProxy' service. These 'Choice' constructs are automatically added by the Workflow Reasoner during the matching stage in Section 5.2. They represent alternative paths that meet the same request requirements. The selection of a specific path is a choice in the composition (visualized on the application portal as a simple selection of payment and delivery method) depending on the developers or the user's preferences. If no specific path is selected, the framework will select one automatically keeping in mind previous executions and presenting to the user the most common one. On the other hand, the user can select a specific delivery possibility, e.g. delivery with payment on delivery, which results in adaptation of the abstract workflow. The Reasoner will remove the 'Choice' construct and the alternative paths resulting in the e-shop graph in Figure 17. This choice is transformed into a rule stating the default service 'DeliveryWithPaymentOnDelivery' to be executed.

\subsubsection{Iterative Pruning}

The Request Operations (Section 4.2) specified in the Request Scheduler define different processing stages (reasoning, mapping, execution, addition of new inputs, reasoning/mapping/execution after manual reconfiguration) of a user's request. These operations are used to iterate over the reasoning, mapping and execution process before the final execution. For example in case the mashup graph is too complex (e.g. having too many branches, conditional paths, decision points) the Execution Engine executes only parts of the mashup. Statefull 
services are not invoked until the final mashup execution in order to preserve the execution state. Only stateless services are evaluated and the intermediary results are stored in the Execution Environment. These are used as additional inputs by the Workflow Reasoner to prune the mashup graph by resolving the decision points or conditional paths.

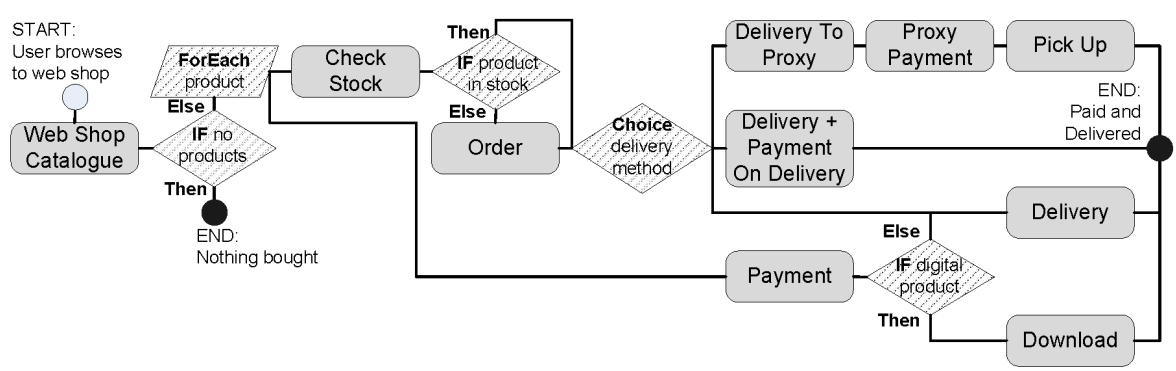

Fig. 18 Automatically constructed e-shop workflow consisting of conditional paths ('ForEach', 'If-Then-Else') and decision points ('Choice').

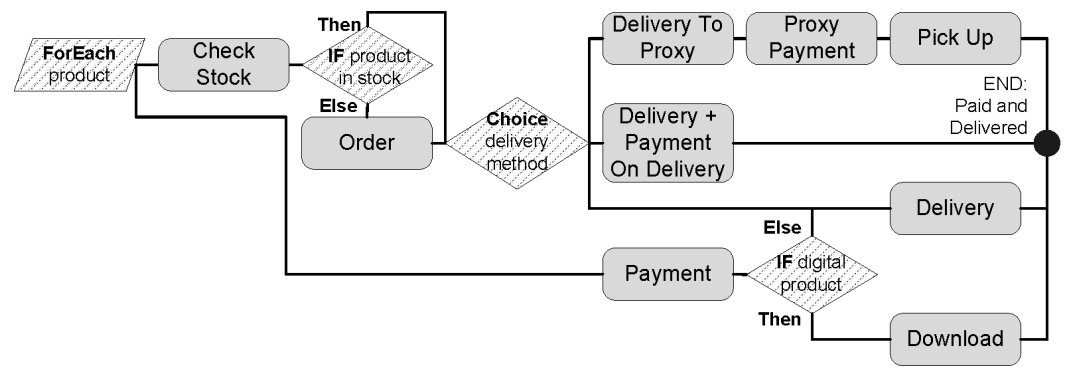

Fig. 19 E-Shop workflow after the selection of products by the user into a shopping cart.

For example, the full mashup workflow as composed by the Workflow Reasoner is illustrated in Figure 18. This graph represents the underlying workflow of an e-shop application. At this point a potential customer browses through the e-shop catalogue and selects interesting products into the shopping cart. If the customer does not proceed with payment, the execution finishes and nothing is bought. Otherwise the 'Web Shop Catalogue' service is executed resulting in Figure 19 with a list of products that if not in stock need to be ordered and after payment delivered to the customer. When presented with the list of selected products, the customer is offered the decision which payment and delivery method he prefers. When one of the three choices is selected (e.g. 'Payment' and 'Delivery'/'Download'), the 'Choice' construct together with the alternative paths are removed leaving the workflow in Figure 20. While payment still needs to be performed by the customer, the rest of the workflow is executed internally. By inspecting the specific type of the products, physical 
or digital, a different workflow remains. Digital products can be downloaded immediately (execution of 'Download') removing the 'Check Stock', 'Order' and 'Delivery' services. Physical products require verifying whether they are in stock or if they need to be ordered (execution of 'Check Stock') before delivery (Figure 21). The idea of pruning is that with bigger mashup graphs, one can choose to execute parts of the workflow, that do not change the state, resulting in additional information that can be used to strip down the mashup to an easier and faster execution.

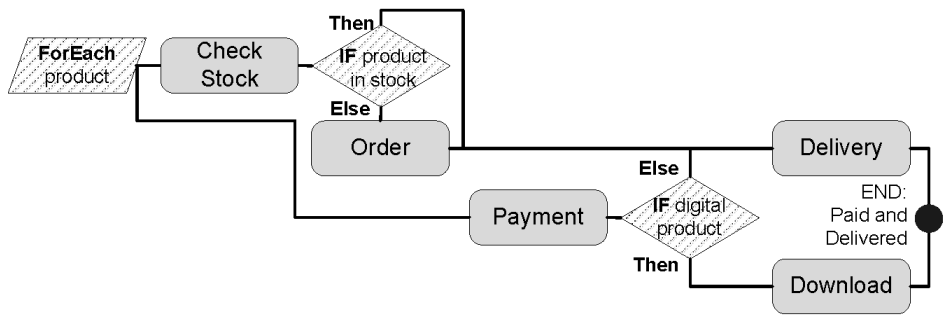

Fig. 20 Adaptation of the e-shop workflow as result of the selection of a specific payment and delivery method and the automatic removal of the alternative paths.

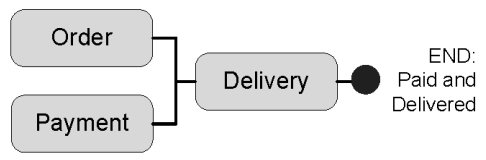

Fig. 21 E-Shop workflow after the determination of the selected products as physical and not in stock.

\subsubsection{Eventing}

The Workflow Reasoner, Service Mapper and Execution Engine are implemented as event listeners subscribed to specific events occurring in the Execution Environment. For example in case of an unavailable service instance, it is added to a taboo list in the Execution Environment and discarded from further use. The Service Mapper subscribes to this event and immediately responds by mapping an equivalent service instance. Table 1 defines the supported events by the Workflow Reasoner, Service Mapper and Execution Engine and their actions.

The eventing mechanism results in a flexible architecture able to support the development of several implementations solving the same user's request, whether it is by extending the three interfaces of the specific modules or combining their functionality in new algorithms. Several Workflow Reasoners can present different service mashups for the same user's request. Parallel working 
Table 1 Supported events and resulting actions by the Workflow Reasoner, Service Mapper and Execution Engine.

\begin{tabular}{|l|l|l|}
\hline Module & Actions & Events \\
\hline Workflow Reasoner & $\begin{array}{l}\text { verifies the consistency of the } \\
\text { graph and reconfigures the } \\
\text { workflow by selecting differ- } \\
\text { ent service nodes or rebuilding } \\
\text { parts it }\end{array}$ & $\begin{array}{l}\text { nescriptions, business logic } \\
\text { rules, deleted/missing ser- } \\
\text { vices/semantic descriptions, } \\
\text { manual service node selection } \\
\text { by the user } \\
\text { Service Mapper }\end{array}$ \\
$\begin{array}{ll}\text { switches between equivalent } \\
\text { nervices with better QoS and } \\
\text { constructs a new executable } \\
\text { process }\end{array}$ & $\begin{array}{l}\text { stances, altered QoS parame- } \\
\text { ters and priorities, reconfigured } \\
\text { semantic mashup by the Work- } \\
\text { flow Reasoner, manual instance } \\
\text { selection by the user, failed } \\
\text { service instance } \\
\text { reconfigured executable pro- } \\
\text { cess by the Service Mapper }\end{array}$ \\
\hline
\end{tabular}

Service Mappers can select service instances favouring various QoS parameters having different weights. A combination of planning and service mapping in one algorithm can optimize both the service mashups graph structure and its global QoS. One can even switch between algorithms in different iteration stages.

\subsubsection{Failure Recovery}

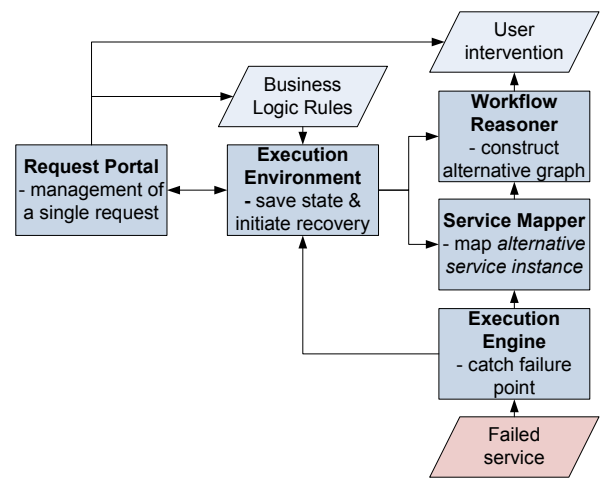

Fig. 22 Recovery from failure using alternative service instances or mashup compositions.

Results from statefull services are also stored in the Execution Environment during mashup execution. If a service fails, this information is used by the Workflow Reasoner and Service Mapper to track the failed points and the current state. The recovery procedure presented in Figure 22 is enhanced with the eventing mechanism automating the selection of alternative equivalent services 
or the partial reconstruction of the workflow. The Execution Engine monitors the mashup execution and intercepts failed service instances. Any intermediary results are saved in the Execution Environment which sends a service failure event to subscribed Service Mappers. The failed service instances are exchanged with equivalent ones still optimizing the global QoS and the Execution Engine resumes with the mashup execution. If there are no equivalent service instances the Execution Environment sends a missing semantic type event to subscribed Workflow Reasoners. The existing mashup is reconfigured by using the current execution state in the Execution Environment to disregard already executed services from the old workflow and constructing an alternative composition for the failed points. After that mapping and execution proceed as usual. In the case that no alternative mashup can be constructed, the Execution Environment presents the user with a semantic description of the failed points clarifying what is expected. The developer can manually reconfigure the mashup using the Frontend Applet (Figure 6), provide additional inputs or add new services. An actual example can be found in Section 6.6 as it is also an evaluation of the performance of the failure procedure.

\section{Framework Evaluation Results}

This section elaborates on the design of an e-shop application developed in collaboration with one of the WTE+ industry partners where all the services are implemented. The selected example validates the optimization details of the presented WTE+ management framework in Section 5 focusing on the comparison between grouping and no grouping of equivalent services into classes during service loading, matching and composition for a pool of 500 services.

Furthermore scalability of the implemented HTN planning algorithm by the Workflow Reasoner (Section 5.3) is evaluated for growing number of services. During mashup composition, three different workflows are compared with service graph: basic, flow control and all. Basic as the name says composes a mashup without the use of control constructs. Flow control automatically adds conditional paths to the mashup workflow depending on the quality of the match between services (Section 5.2). All adds decision points also referred as 'Choice' constructs to the workflow path allowing during execution the user to select a specific execution path or the automatic selection of a random path by the framework. These experiments present actual expansion of the service mashup first without control constructs, then with conditional paths, and at then with decision points.

Last measure is the construction of the executable process by the Service Mapper and the obtained QoS results of the late binding for different financial and time parameter priorities.

Section 6.6 on adaptation evaluates the time loss while reconfiguring the service mashup during failure (Section 5.4.4). It illustrates the reduction of effort compared to a semi-automatic service composer, or BPEL manual composition which cannot be automatically reconfigured. 
6.1 Use case description

Shopping on the Internet is a booming business consisting of small e-shops, ran by just one person, second hand e-shops, and large competitors like Amazon. Amazon started as a book shop and has grown into a multimillion corporation in just several years. Other services supporting these online shopping activities are the hundreds of payment and shipping services. The best known payment service is PayPal, bought by the largest online second hand store, eBay. Companies like UPS, DHL and FedEx offer shipping services that can be ordered and tracked online.

This section describes functionality provided by such an e-shop application constructed by the WTE+ management framework involving an e-commerce industry partner. For this purpose an e-shop ontology was created used for the enrichment of the implemented 500 e-shop services with OWL-S descriptions.

\subsubsection{Design of an E-Shop Application}

A sale consists of a customer buying one or more products. Traditionally, this means that:

Trigger: A potential customer browses the product catalogue of the e-shop.

Initial state: The e-shop and customer info is known. This includes account information necessary to make payments to the e-shop.

Goal description: The composition is successfully executed, when the following effects are reached:

1. The customer ordered the product(s).

2. The customer paid for the product(s).

3. The product(s) was(were) delivered to the customer.

- Digital products, such as music and software, are downloaded.

- Physical products are transported to the customer's delivery address or to a proxy point of the customer's choice.

\subsubsection{Semantic Description of E-Shop Services}

An e-shop ontology is created using the Protégé Editor defining the required e-shop concepts. The constructed e-shop ontology in Figure 23(a) presents the necessary taxonomy for the evaluated use case. It consists of customer account information, details on the available proxy points and the obtainable products divided into digital and physical, whether in stock or not. The relationships between two OWL concepts are defined as object properties in Figure 23(b) used for the definition of SWRL rules as conditions. An example condition can be the delivery of a digital product to the customer.

For testing purposes, several e-shop services are enriched with OWL-S descriptions generated automatically using the OWL-S API. XSL transformations are added manually to translate between the e-shop ontology and the XSD (XML Schema Definition) data types used in the WSDL interface. The 


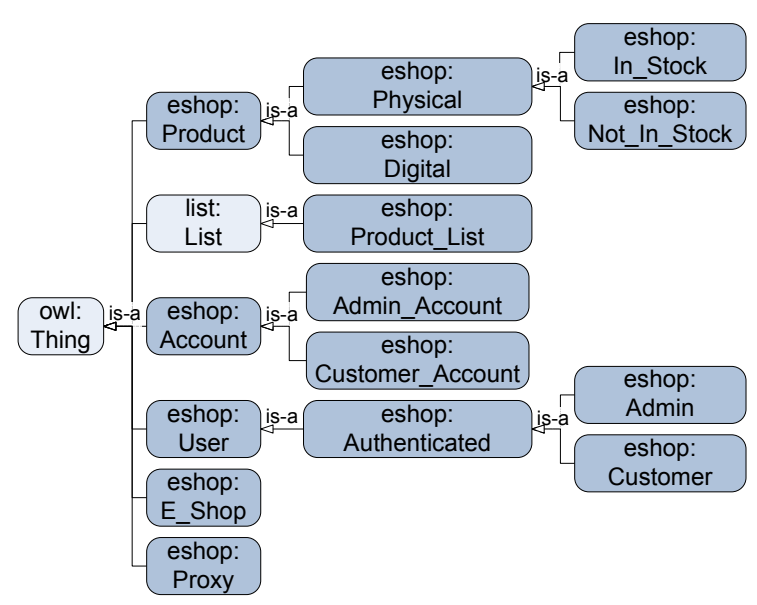

(a) E-Shop ontology

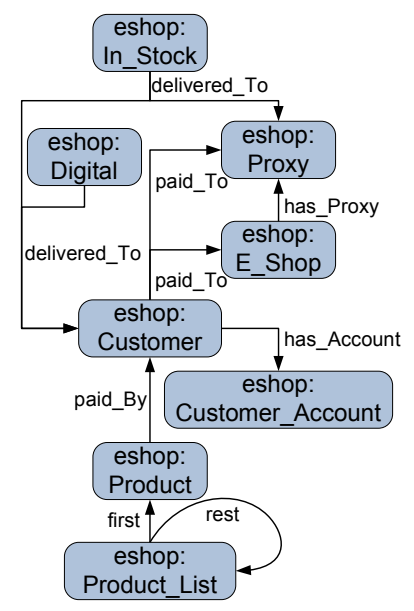

(b) E-Shop properties

Fig. 23 Defined e-shop concepts and relationships between them.

e-shop services are assigned QoS parameters (execution time and cost) with different weights used by the Service Mapper for selecting service instances corresponding to specific QoS requirements.

6.2 Experiment Setup Description

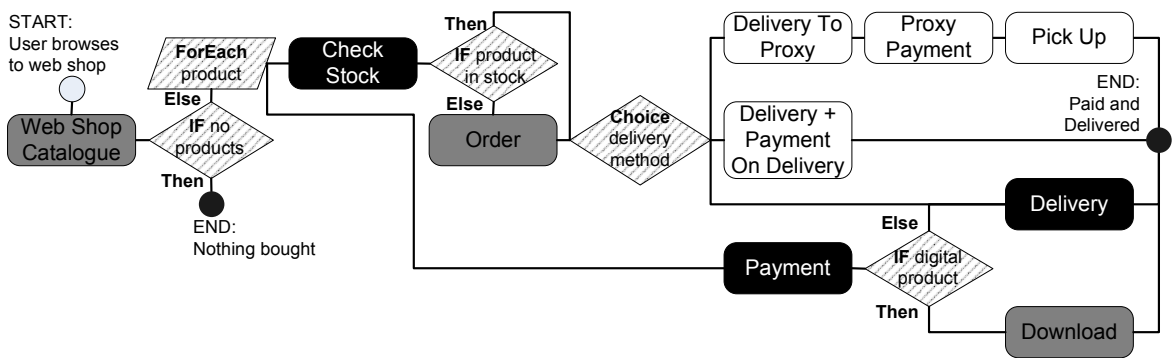

Fig. 24 E-Shop workflow consisting of black services (Basic Reasoner without the use of control constructs), grey services (Flow Control Reasoner adding control constructs such as 'ForEach', 'IfThenElse'), and white services (Reasoner using the 'Choice' construct where unlike the other Reasoners alternative paths are also expanded)

For testing purposes, the e-shop mashup in Figure 24 was designed, consisting of 6 levels, breadth of maximum 3 services, and 10 different available service nodes multiplied by 50 semantically equivalent services per node. In order to evaluate the semantic reasoning, different measurements were observed: the initial classification of service instances into groups of equivalent semantic 
descriptions, semantic matching of services to a required interface, the construction of a semantic mashup by the Workflow Reasoner, the selection of a specific service instance by the Service Mapper providing for an optimum QoS, the construction of a executable process by the Service Mapper, and the runtime recovery during failure. The measurements were performed on the iLab.t Virtual Wall (iLab.t Virtual Wall 2011) which is a large scale generic test environment for advanced network, distributed software and service emulation and evaluation, and supports scalability research. The Virtual Wall facilities consist of 100 nodes (dual processor, dual core servers, 6x1 Gb/s interfaces per node) interconnected via a non-blocking $1.5 \mathrm{~Tb} / \mathrm{s}$ VLAN Ethernet switch, and a display wall (20 monitors) for experiment visualization. Each server is connected with 4 or $6 \mathrm{~Gb}$ Ethernet links to the switch.

In order to evaluate the QoS-aware late binding algorithm of the Service Mapper the service instances are assigned two kinds of QoS parameters: execution time and financial cost. The time parameters exhibit a Gaussian distribution (mean 50, standard deviation 17), resulting in most of the services having an average time and a few faster or slower exceptions. As we expect both parameters (time and cost) to be intertwined, the time distribution corresponds to two overlapping Gaussian distributions (mean 30 and 70, standard deviation 10) of the cost parameter. The impact of this method is that on average, fast services are as expected more expensive than slower ones, but exceptions like fast and rather cheap services do exist. These QoS parameters are assigned different priorities by adding a weight factor (time-cost: 1-3, 1-2, $2-3,1-1,3-2,2-1,3-1)$ to the QoS Value used by the QoS Comparator during service instance selection.

\subsection{Grouping of Equivalent Semantic Types}

The service loading time into the repository is evaluated for growing number of equivalent services with or without service grouping detailed in Section 5.1. The results are presented in Figure 25 for a pool of 500 services classified into 10 groups of 50 equivalent services. During the loading of the first service description, several other ontologies need to be loaded like the OWL-S Profile, Process, and Grounding ontologies, the use case specific e-shop ontology, the SWRL rules ontologies. This is a one-time-only action during the loading of the first service description that takes up on average $410 \mathrm{~ms}$ with a standard deviation of $35 \mathrm{~ms}$. Once this is done, only the loading of the OWL-S service descriptions into the repository and additionally for grouping the time needed for the semantic comparison of the service interfaces classifying them into equivalent service groups is taken into account. Although the difference between service loading with or without grouping, resp. 5 and 1 minute(s) for 500 services, grows steadily with growing number of services, it is a necessary first time comparison that considerably speeds up the service matching process during mashup construction by the Workflow Reasoner as can be seen in Section 6.4. 


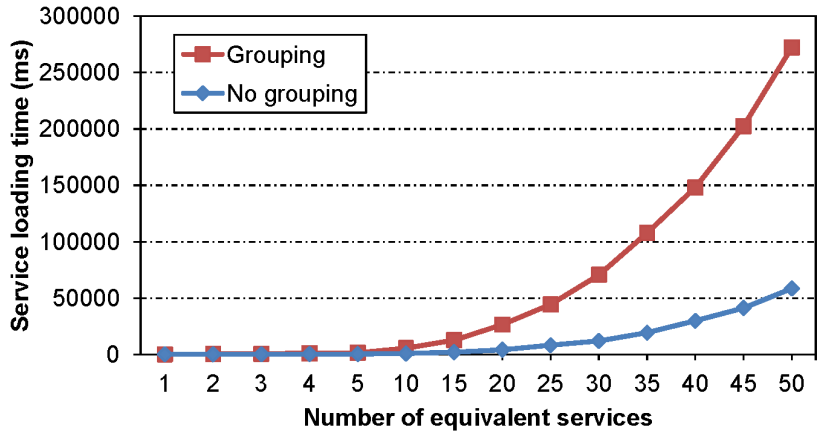

Fig. 25 Comparison of the service loading time for growing number of e-shop services with or without service grouping.

\subsection{Mashup Creation}

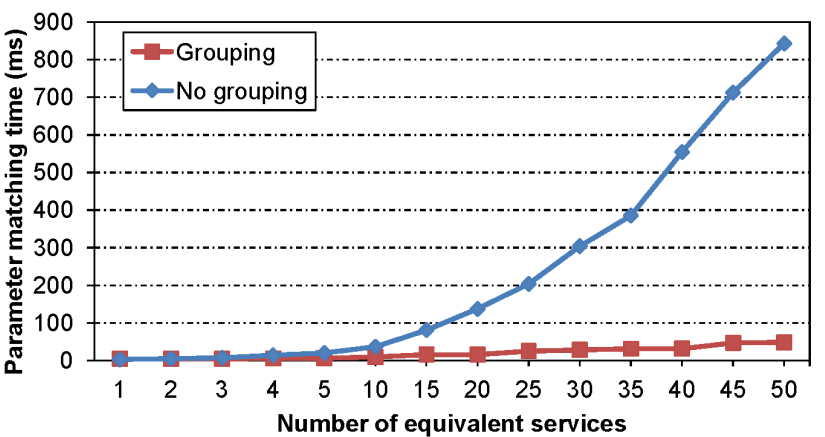

Fig. 26 Comparison service querying for a matching parameter for growing number of e-shop services with or without service grouping.

For the evaluation of the Workflow Reasoner the user's request 'payment and delivery of several products to a customer' is defined. During construction of a service mashup resolving this goal services are queried matching required inputs and conditions as described in Section 5.3. Figure 26 and Figure 27 present the querying for a respectively required service input and condition from the existing service pool with or without service grouping. Logically, with grouped services, one only needs to consider the 10 service groups while without grouping all services need to be queried resulting in an exponential increase of service matching. It should be noted that querying for an input is much faster (1 second without and $50 \mathrm{~ms}$ with grouping) than for a condition (1 minute without and 1 second with grouping) for a pool of 500 services. This is due to the fact that for input matching only 2 parameters are compared while for condition matching 2 pairs of parameters and the properties between them are compared. Although in comparison to the exponentially growing 


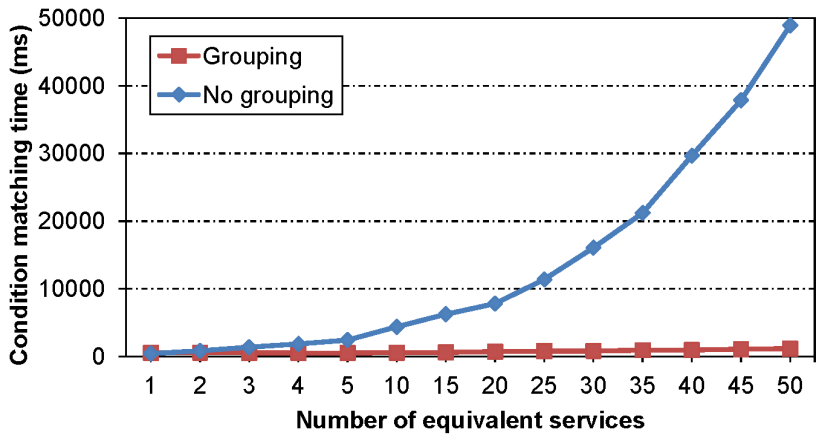

Fig. 27 Comparison service querying for a matching condition for growing number of e-shop services with or without service grouping.

without grouping scenario, grouping presents a even solution there is still a small overhead to be seen due to the specific software implementation. This is the result of the memory usage while querying each class consisting of a list of equivalent services.

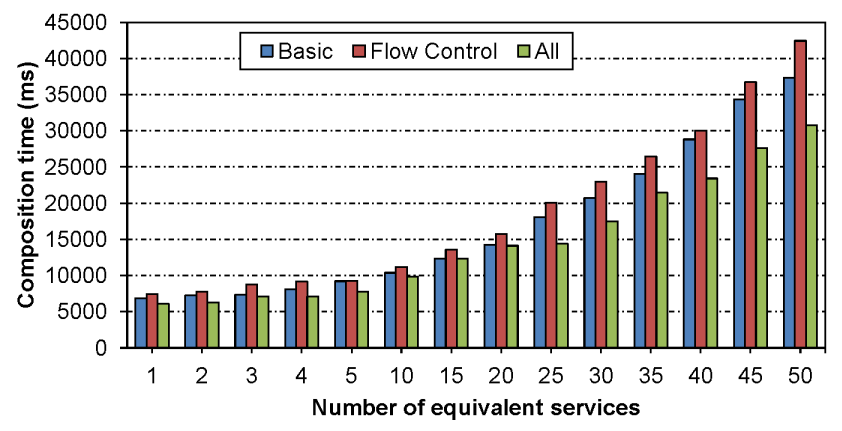

Fig. 28 Composition time with growing reasoning complexity (Basic - Flow Control - All) as a function of the number of services for the grouped service pool.

The limited growth becomes more visible in the accumulated result of the service composition algorithm in Figure 28. It presents the evaluation of the Workflow Reasoner for up to 500 or also 50 equivalent services with service grouping. Distinction is made between a basic Reasoner without the use of control constructs (Basic), a Reasoner adding flow controls like 'ForEach', 'IfThenElse' (Flow Control) and finally a Reasoner using the 'Choice' construct where unlike the other reasoners alternative non-equivalent services are also expanded (i.e. delivery at home, pick up from proxy) (All). These solutions construct different service mashup graphs consisting of 3 (Basic), 6 (Flow Control) and 10 (All) service nodes (Figure 24). The composition time is an accumulation of querying for matching service inputs and conditions, the actual construction of the mashup graph through linking of the matching services 


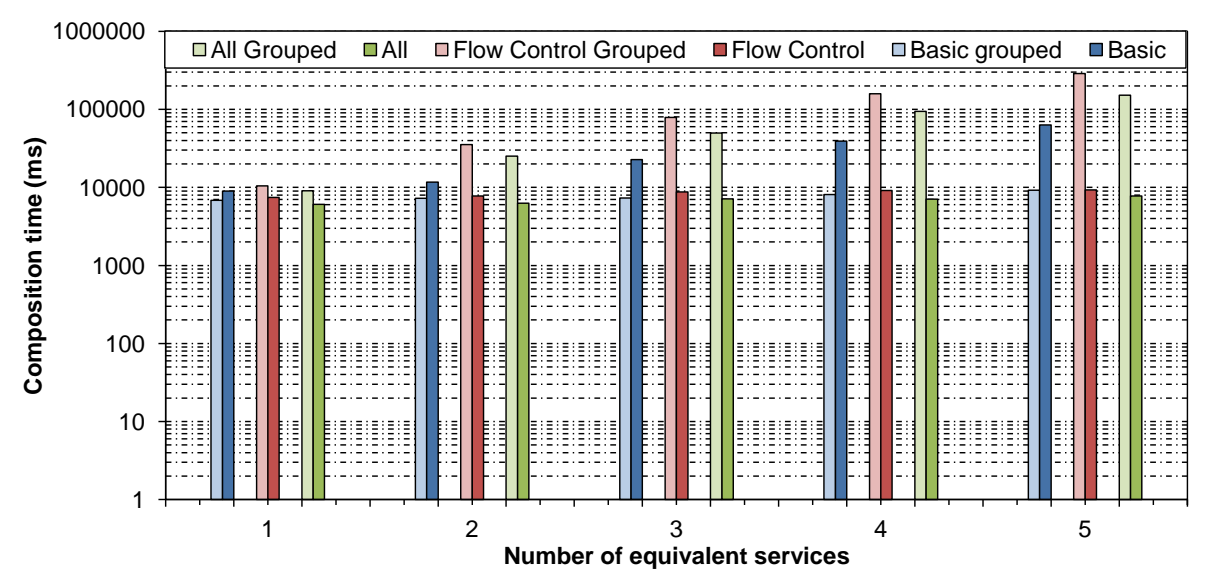

Fig. 29 Comparison of the composition time with growing reasoning complexity as a function of the number of services.

to each other and removing of partially constructed alternative paths (expect for the All algorithm where alternatives are kept). From the figure it is visible that the Flow Control algorithm is the slowest due to the fact the it provides a correct solution through the addition of conditional paths which are discarded by the limited reasoning of the Basic algorithm. The All algorithm also keeps track of these conditional solutions but as it does not remove alternative paths like the other two it is the fastest of the three algorithms. Although due to the software implementation the composition time goes up to 1 minute for 500 services, it is a good optimization in comparison to the without grouping case as can be seen in Figure 29 for just 5 equivalent services. With grouping it varies between 8 and 10 seconds with standard deviation between 100 and 300 milliseconds as only the groups of equivalent services are considered while without grouping all available services are queried resulting in exponentially growing composition time.

\subsection{QoS-aware Mapping of Service Instances}

During the mapping phase for each semantic node of the constructed mashup a service instance is selected from the pool of equivalent services. In case of one-to-one mapping there is evidently no time loss, which is the case without service grouping. Even with grouping there is an almost insignificant increase, between 2 to 5 milliseconds for 5 equivalents, in service selection time.

As mentioned before for the evaluation of the QoS-aware service selection of the Service Mapper the service instances are assigned two kinds of QoS parameters: execution time (Gaussian distribution, mean 50, standard deviation 17) and financial cost (Gaussian distribution, mean 30 and 70, standard deviation 10). These QoS parameters are assigned different priorities by adding a weight factor (time-cost: 1-3, 1-2, 2-3, 1-1, 3-2, 2-1,3-1) to the QoS Value 


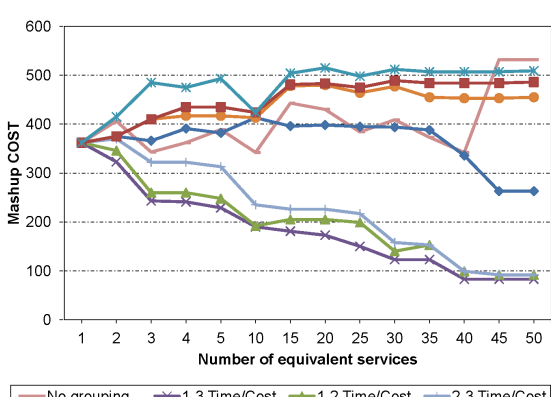

-No grouping $\approx-1-3$ Time/Cost $\rightarrow-1-2$ Time/Cost $\div-2-3$ Time/Cost

(a) Mashup execution cost.

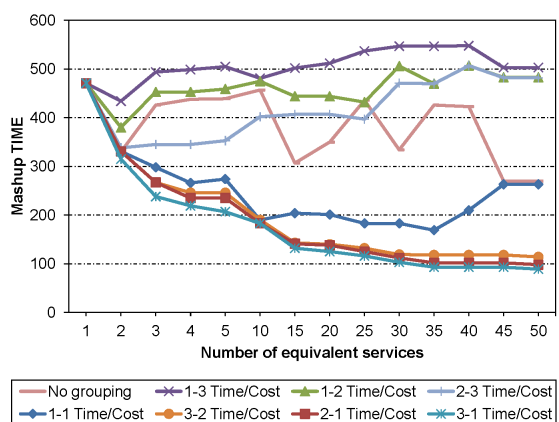

(b) Mashup execution time.

Fig. 30 Comparison of the impact of the service QoS parameters during service selection on the total mashup execution cost and time for different cost and time priority in function of the number of services.

used by the QoS Comparator during service instance selection. In Figure 30 the total cost and time of the complete e-shop mashup are compared for the case without and with grouping for these cost and time priorities. Without grouping, random services are selected by the Workflow Reasoner (e.g. always the new pool) which results in a rather high mashup cost and time. With grouping, the Service Mapper selects a service from a group of equivalents by comparing their time and cost parameters using the weight factors (adjusted as preferred during request configuration). Once the number of equivalent services grows the mashup cost and time both drop respectively in Figure 30(a) and Figure 30(b) for high priorities. The following observations can be made:

- As expected the different curves are all neatly ordered depending on the parameter priority.

- The higher the priority of one of the parameters, the worser mashup QoS for the other resulting in a curve above the random one of the without grouping example.

- For growing number of services the mashup QoS values for both parameters sometimes tend to increase even for high priorities. For this specific example in Figure 30(a) for 35 equivalents and 1-2 Time/Cost priority ratio the cost increases while in Figure 30(b) the time drops. This is explained by the fact the some services have very low time parameters which results in the sudden mashup time drop that cannot even be beaten by a slightly higher cost priority. In this way the Service Mapper favours a specific QoS parameter but still takes into account dominant QoS Values of other parameters.

- In contrast with the time curve in Figure 30(b) that is always under the without grouping case for the of 1-1 Time/Cost priority ratio the cost in Figure 30(a) not even does not exhibit an equal drop but it goes above it for some service pools. The explanation is that in the new service pool services with very low time parameter have rather high cost parameter as result of the specific assignment of the QoS weights. As the time weight 
has a Gaussian distribution with mean 50 and low time values correspond to Gaussian cost distribution with mean 70, the mashup cost cannot drop equally to the mashup time drop.

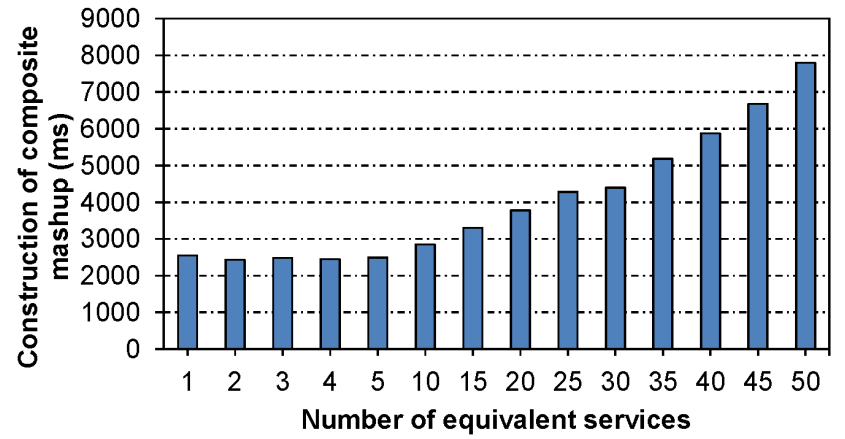

Fig. 31 Construction of a composite mashup from the service graph including service selection based on QoS as function of the number of services.

The service selection overhead for growing number of equivalent services is presented in Figure 31. It represents the construction of an executable composite process from the created mashup graph by the All Workflow Reasoner algorithm for grouped service instances. The measurements without grouping are equivalent to the solution of 1 service per equivalent class on the figure. The executable process construction time consists of the described above QoSaware service instance selection, the creation of service bindings required for the linking of service results to service inputs during execution, and the addition of control constructs. The maximum 1 second increase for each new pool of 50 services of the construction time is due to the growing service pool as the mashup graph is always the same.

\subsection{Dynamic Mashup Adaptation}

As described in Section 5.4 during execution the state is saved in case of a resource or service failure. Figure 32 details the at-runtime eventing mechanism during recovery including the different steps (composition, mapping, execution) taken by the framework and the time for executing each step. A basic workflow required for product payment and delivery is presented in Figure 33(a). Simultaneously to the execution of the product 'Payment', the 'CheckStockAndOrder' service fails. The Service Mapper selects an equivalent service instance replacing the failed one (Case (b) in the figure). Afterwards the Execution Engine carries on the execution while avoiding repeated 'Payment' execution. The time loss in respect to no failure (Case (a) in the figure) is insignificant. On the other hand, if no equivalent service instance is found (Case (c) in the figure), the Workflow Reasoner reconfigures the original mashup 


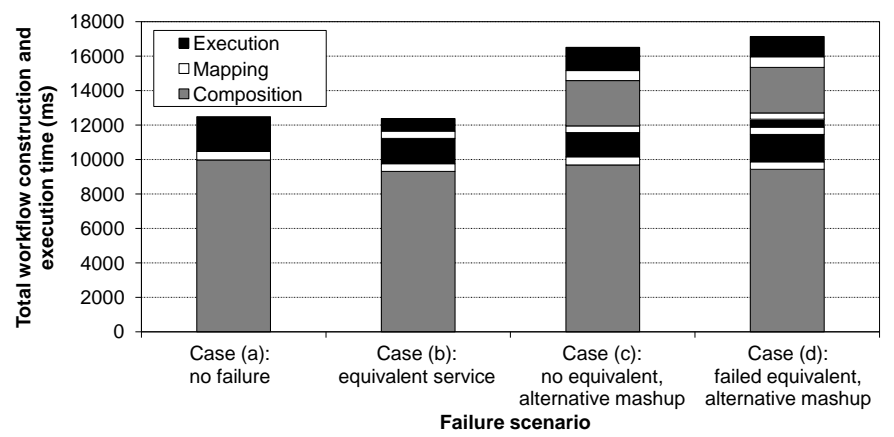

Fig. 32 Impact of dynamic reuse of results, state and eventing on the mashup composition, mapping and execution time during service failure. In Case (a) no failure occurs. In Case (b) a failed instance is replaced by an equivalent one by the Service Mapper. In Case (c) there are no equivalent instances so the Workflow Reasoner reconfigures the mashup with alternative path. In Case (d) both the equivalent instances fail before going back to the Workflow Reasoner.

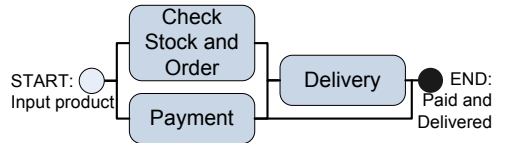

(a) Workflow before failure.

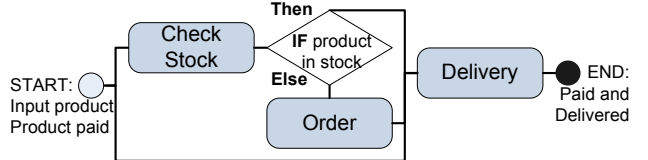

(b) Workflow after service failure.

Fig. 33 Constructed workflow before failure 33(a) and after failure 33(b) with alternative composition by the Workflow Reasoner.

constructing an alternative solution as seen in Figure 33(b), treating the 'Payment' requirements as already met and thus as part of the initial state. In case of no equivalents, this adds no more than 5 seconds. With each failed equivalent service half a second is lost (Case (d) in the figure) as the Service Mapper and consequently the Execution Engine first go through all equivalents before returning to the Workflow Reasoner.

\section{Conclusions}

This paper presents the design and validation of the WTE+ management framework supporting automatic composition, execution and dynamic adaptation of custom-made service mashups. It disposes of a user interface for the management of semantically annotated services and the definition of users' requests (req. 1). Planning algorithms are implemented that automatically assemble service mashups out of existing semantically enriched services (req. 2) resolving users' requests. Novelty with respect to the presented related work is the automatic addition of control constructs depending on the on the quality of the semantic match between the available services. The framework is optimized with late binding to actual service instances satisfying QoS constraints 
and requirements (req. 3). Dynamic at-runtime adaptation of the constructed mashups is achieved as result to changing context such as new services, new business logic, failure or overload of network elements through dynamic reconfiguration and personalization of the constructed mashups (req. 5). A realistic e-shop application is implemented illustrating and evaluating the workflow execution optimizations in close collaboration with an e-commerce WTE+ project partner.

The increasing number of services available on the Web and within enterprises results in performance and scalability demands of the designed planning algorithms. These quality attributes are strongly dependent on the classification of the semantically equivalent service instances (req. 4). As the presented Workflow Reasoner only considers semantic types (representing a group of equivalent services) there is no time loss during workflow planning with growing number of equivalent services. The Service Mapper presents a trade-off between various QoS parameters favouring one or more of them through the use of weight factors (req. 3). The WTE+ management framework supports application managers with the automatic construction of custom-made service mashups at a minimum performance cost without the need for constant IT intervention.

On an architectural level the WTE+ management framework consists of a monitoring interface generating logging information on the different steps and results from the mashup construction and execution process (req. 7). It comes with an interface presenting in a graphical way the constructed mashup graph and the selected resources for execution offering domain experts the possibility for manual tuning of the graph. The implemented QoS-aware planning algorithms and execution engines are implemented as plug-ins enabling the easy extension of their respective interfaces with new implementations (req. 6).

Future work includes (i) the extension of the planning and execution framework with a distributed deployment making optimal use of the available resources for the execution of the service instances and (ii) the study of techniques for automatic extraction of new knowledge from request patterns, behaviour and feedback of users for the optimization of future requests.

\section{Summary Points}

Available functionality as a starting point:

- Web services as reusable components in Service-Oriented Architectures.

- Computer interpretable Web services thanks to the use of ontologies and semantic languages like OWL-S.

- Matching a service output to a service input when similar semantic concepts are represented.

- Execution system for semantically annotated Web services.

Novel functionality supported by the WTE+ management framework:

- User interface managing the semantically annotated services and the definition of users' requests (Section 4.1). 
- Enrichment of services with semantic descriptions and automated construction of mashups performed in OWL-S adopting the richness of the language characteristics such as control constructs ('Split+Join', 'IfThenElse', 'ForEach', 'Choice') (Section 5.1.2, 5.3).

- Automated addition of control constructs depending on the quality of the match between the individual services during the composition process resulting in complex service workflows (Section 5.2).

- Dynamic adaptation to changing runtime environment through the monitoring and updating of service QoS parameters (Section 5.1.1, 5.4).

- Mashup optimization due to partial workflow execution feeding back intermediary results resulting in reconfiguration of the composition (Section 5.4.2).

- Request personalization through user-defined business logic rules (Section 5.4.1).

- Plug-in design of the overall framework allowing for future additions of new QoS-aware planning algorithms and execution engines (Section 4.2, 5.4.3).

Acknowledgements Anna Hristoskova would like to thank the Special Research Fund of Ghent University (BOF) for financial support through her PhD grant. This work is partly funded by WTE+ (WTE+ 2008-2010), an IBBT (Interdisciplanary Institute for BroadBand Technology) GBO (Joint Basic Research) project on the definition of an open architecture that allows the creation, sharing and composition of service mashups, seamlessly combining functionality available on the Web, the enterprise or the telecom operator.

\section{References}

Agre, G., Marinova, Z.: An infrawebs approach to dynamic composition of semantic web services. Cybernetics and Information Technologies 7(1):45$61(2007)$

Akkiraju, R., Farrell, J., Miller, J., Nagarajan, M., Schmidt, M.T., Sheth, A., Verma, K.: WSDL-S, Web Service Semantics. (2005). Available from http://www.w3.org/Submission/WSDL-S/

Ardagna, D., Comuzzi, M., Mussi, E., Pernici, B., Plebani, P.: PAWS: A Framework for Executing Adaptive Web-Service Processes. IEEE Software, 24(6):39-46 (2007)

Aschoff, R.R., Zisman, A.: QoS-driven Proactive Adaptation of Service Composition. In: International Conference on Service Oriented Computing (ICSOC 2011), (2011)

Avellino, G., Boniface, M., Cantalupo, B., Ferris, J., Matskanis, N., Mitchell, B., Surridge, M.: A dynamic orchestration model for future internet applications. Towards a Service-Based Internet, pp 266-274 (2008).

Baresi, L., Di Nitto, E., Ghezzi, C., Guinea, S.: A Framework for the Deployment of Adaptable Web Service Compositions. Service Oriented Computing and Applications Journal 1(1):75-91 (2007)

Baresi, L., Guinea, S.: Consumer Mashups with Mashlight. Towards a ServiceBased Internet, Proceedings of ServiceWave 2010, 6481:112-123 (2010) 
Battle, S., Bernstein, A., Boley, H., Grosof, B., Gruninger, M., Hull, R., Kifer, M., Martin, D., McIlraith, S., McGuinness, D., Su, J., Tabet, S.: SWSO, Semantic Web Services Ontology. (2005). Available from http://www.w3.org/Submission/SWSF-SWSO/

Beisiegel, M., Blohm, H., Booz, D., Dubray, J., Colyer, A., Edwards, M., Ferguson, D., Flood,B., Greenberg, M., Kearns, D., et al: Service Component Architecture. Building Systems using a Service Oriented Architecture. BEA, IBM, Interface21, IONA, Oracle, SAP, Siebel, Sybase, white paper, version 09 edition (2005)

Berbner, R., Spahn, M., Repp, N., Heckmann, O., Steinmetz, R.: Heuristics for QoS-aware Web Service Composition. In: IEEE International Conference on Web Services, pp 72-82 (2006)

Berners-Lee, T., Hendler, J., Lassila, O.: The semantic web: A new form of web content that is meaningful to computers will unleash a revolution of new possibilities. Journal of the Scientific American 284(5):34-43 (2001)

Bertoli, P., Pistore, M., Traverso, P.: Automated composition of Web services via planning in asynchronous domains. Artificial Intelligence 174(3-4):316 361 (2010)

Brogi, A., Canal, C., Pimentel, E., Vallecillo, A.: Formalizing web service choreographies. Electronic Notes in Theoretical Computer Science 105(10):73-94 (2004)

Cândido, G., Barata, J., Colombo, A.W., Jammes, F.: Soa in reconfigurable supply chains: A research roadmap. Engineering applications of artificial intelligence 22(6):939-949 (2009)

Canfora, G., Di Penta, M., Esposito, R, Villani, M.L.: QoS-Aware Replanning of Composite Web Services. In: IEEE International Conference on Web Services, pp 121-129 (2005)

Carreras, I., Chlamtac, I., Pellegrini, FD., Miorandi, D.: Bionets: Bio-inspired networking for pervasive communication environments. IEEE Transactions on Vehicular Technology 56(1):218-229 (2007)

Dai, Y., Yang, L., Zhang, B.: QoS-Driven Self-Healing Web Service Composition Based on Performance Prediction. Journal of Computer Science and Technology, 24(2):250-261 (2009)

Daniel, F., Casati, F., Benatallah, B., Shan, M.C.: Hosted Universal Composition: Models, Languages and Infrastructure in mashArt. Conceptual Modeling-ER 2009, pp 428-443 (2009)

Daniel, F., Soi, S., Tranquillini, S., Casati, F., Heng, C., Yan, L.: From people to services to ui: Distributed orchestration of user interfaces. Proceedings of Business Process Management(BPM'10), 6336:310-326 (2010)

Di Nitto, E., Ghezzi, C., Metzger, A., Papazoglou, M., Pohl, K.: A journey to highly dynamic, self-adaptive service-based applications. Automated Software Engineering 15(3):313-341 (2008)

Fujii, K., Suda, T.: Semantics-based dynamic web service composition. International Journal of Cooperative Information Systems 15(3):293-324 (2006)

Gamha, Y., Bennacer, N., Romdhane, L.B., Vidal-Naquet, G., Ayeb, B.: A statechart-based model for the semantic composition of web services. In: 
Fourth International Workshop on Semantic Web for Services and Processes (SWSP 2007), pp 49-56 (2007)

Gubala, T., Herezlak, D., Bubak, M., Malawski, M.: Semantic composition of scientific workflows based on the petri nets formalism. In: Proceedings of the Second IEEE International Conference on e-Science and Grid Computing (e-Science 2006), pp 12-19 (2006)

Guinea, S., Kecskemeti, G., Marconi, A., Wetzstein, B.: Multi-layered Monitoring and Adaptation. In: International Conference on Service Oriented Computing (ICSOC 2011), (2011)

Hamadi, R., Benatallah, B.: A petri net-based model for web service composition. In: Proceedings of the 14th Australasian database conference (ADC2003), pp 191-200 (2003)

Hausheer, D., Nikander, P., Fogliati, V., Wünstel, K., Callejo, M., Jorba, S., Spirou, S., Ladid, L., Kleinwägchter, W., Stiller, B., et al: Future Internet Socio-Economics-Challenges and Perspectives. Towards the Future Internet pp 1-11 (2009)

Hawke, S., Herman, I., Prud'hommeaux, E., Swick, R.: Semantic Web, Providing a common framework that allows data to be shared and reused across application, enterprise, and community boundaries. (2011). Available from http://www.w3.org/2001/sw/

Horrocks, I., Patel-Schneider, P.F., Boley, H., Tabet, S., Grosof, B., Dean, M.: SWRL: A Semantic Web Rule Language Combining OWL and RuleML. (2004). Available from http://www.w3.org/Submission/SWRL/

Hristoskova, A., Volckaert, B., De Turck, F.: Dynamic composition of semantically annotated web services through qos-aware htn planning algorithms. In: Proceedings of the Fourth International Conference on Internet and Web Applications and Services (ICIW 2009), pp 377-382 (2009)

Hristoskova, A., Moeyersoon, D., Van Hoecke, S., Verstichel, S., Decruyenaere, J., De Turck, F.: Dynamic composition of medical support services in the ICU: Platform and algorithm design details. Computer Methods and Programs in Biomedicine 100(3):248-264 (2010)

iLab.t Virtual Wall. (2011). Available from http://ilabt.ibbt.be/

Klusch, M., Gerber, A., Schmidt, M.: Semantic web service composition planning with owls-xplan. In: Proceedings of the First International AAAI Fall Symposium on Agents and the Semantic Web pp 55-62 (2005)

Klusch, M., Fries, B., Sycara, K.: OWLS-MX: A hybrid Semantic Web service matchmaker for OWL-S services. Web Semantics: Science, Services and Agents on the World Wide Web 7(2):121-133 (2009)

Lara, R., Roman, D., Polleres, A., Fensel, D.: A conceptual comparison of WSMO and OWL-S. Web Services, pp 254-269 (2004)

Lecue, F., Delteil, A., Leger, A.: Towards a semantic state transition system for automated generation of data flow in web service composition. In International Journal of Semantic Computing 3(4):499-526 (2009)

Leitner, P., Michlmayr, A., Rosenberg, F., Dustdar, S.: Monitoring, Prediction and Prevention of SLA Violations in Composite Services. In: IEEE International Conference on Web Services, pp 369-376 (2010) 
Lin, K.J., Zhang, J., Zhai, Y., Xu, B.: The Design and Implementation of Service Process Reconfiguration with End-to-end QoS Constraints in SOA. Journal of Service Oriented Computing and Applications 4:1-12 (2010)

Liu, J., Cui, J., Gu, N.: Composing web services dynamically and semantically. In: IEEE International Conference on E-Commerce Technology for Dynamic E-Business (CEC-East'04), pp 234-241 (2004)

Martin, D., Burstein, M., Hobbs, J., Lassila, O., McDermott, D., McIlraith, S., Narayanan, S., Paolucci, M., Parsia, B., Payne, T., Sirin, E., Srinivasan, N., Sycara, K.: OWL-S, Semantic Markup for Web Services. (2004). Available from http://www.w3.org/Submission/OWL-S/

Metzger, A., Sammodi, O., Pohl, K., Rzepka, M.: Towards Pro-active Adaptation with Confidence Augmenting Service Monitoring with Online Testing. In: ICSE Workshop on Software Engineering for Adaptive and SelfManaging Systems (SEMAS 2010), pp 20-28 (2010)

Miyagi, M., Ohkubo, K., Kataoka, M., Yoshizawa, S.: Performance Prediction Method for Web-Access response Time Distribution Using Formula. In: Network Operations and Management Symposium (NOMS 2004) 1:905906 (2004)

Möller, T.: OWL-S API. (2010). Available from http://on.cs.unibas.ch/owlsapi/index.html

Papazoglou, M.P., Traverso, P., Dustdar, S., Leymann, F.: Service-oriented computing: A research roadmap. International Journal of Cooperative Information Systems 17(2):223-255 (2008)

Peltz, C.: Web services orchestration and choreography. Computer, pp 46-52 (2003)

Pistore, M., Marconi, A., Bertoli, P., Traverso, P.: Automated Composition of Web Services by Planning at the Knowledge Level, In: International Joint Conference on Artificial Intelligence (2005)

Pfeffer, H.: A underlay system for enhancing dynamicity within web mashups. International Journal On Advances in Software 2(1):63-75 (2009)

Pietschmann, S.: A model-driven development process and runtime platform for adaptive composite web applications. International Journal On Advances in Internet Technology 2(4):277-288 (2009)

Prete, L.D., Capra, L.: Mosca: seamless execution of mobile composite services. In: Proceedings of the 7th Workshop on Reflective and Adaptive Middleware pp 5-10 (2008)

Roman, D., Keller, U., Lausen, H., de Bruijn, J., Lara, R., Stollberg, M., Polleres, A., Feier, C., Bussler, C., Fensel, D.: Web service modeling ontology. Applied Ontology 1(1):77-106 (2005)

Sirin, E., Parsia, B., Hendlera, J.: Filtering and selecting semantic web services with interactive composition techniques. IEEE INTELLIGENT SYSTEMS 19(4):42-49 (2004)

Sirin, E., Parsia, B., Wu, D., Hendler, J., Nau, D.: Htn planning for web service composition using shop2. Journal of Web Semantics 1(4):377-396 (2004)

Stanford University: Protégé. (2011). Available from http://protege.stanford.edu/ 
Taivalsaari, A., Mikkonen, T.: Mashups and modularity: Towards secure and reusable web applications. 23rd IEEE/ACM International Conference on Automated Software Engineering-Workshops pp 25-33 (2008)

Tosi, D., Denaro, G., Pezz, M.: Towards autonomic service-oriented applications. International Journal of Autonomic Computing 1(1):58-80 (2009)

Tsalgatidou, A., Athanasopoulos, G., Pantazoglou, M., Pautasso, C., Heinis, T., Grønmo, R., Hoff, H., Berre, A.J., Glittum, M., Topouzidou, S.: Developing scientific workflows from heterogeneous services. ACM Sigmod Record 35(2):22-28 (2006)

Valle, M., Ramparany, F., Vercouter, L.: Dynamic service composition in ambient intelligence environments: a multi-agent approach. In: Proceeding of the First European Young Researcher Workshop on Service-Oriented Computing (2005)

Van Der Aalst, W., Dumas, M., Ter Hofstede, A.: Web service composition languages: old wine in New bottles?. In: Proceeding of the 29th EUROMICRO Conference: New Waves in System Architecture, IEEE, pp 298-305 (2003)

Verma, K., Sheth, A.: Semantically annotating a web service. IEEE Computer Society 11(2):83-85 (2007)

WTE+, Moving beyond Web/Telco/Enterprise 2.0. (2008-2010). Available from https://projects.ibbt.be/wteplus/

Yelmo, J.C., Trapero, R., del Álamo, J.M., Siene, J., Drewniok, M., Ordás, I., McCallum, K.: User-driven service lifecycle management - adopting internet paradigms in telecom services. Lecture Notes in Computer Science 4749:342$352(2009)$

Zhang, S., Zong, Y., Ding, Z., Liu, J.: Workflow-oriented grid service composition and scheduling. International Conference on Information Technology: Coding and Computing (ITCC'05) 2:214-219 (2005)

Zisman, A., Spanoudakis, G., Dooley, J.: A Framework for Dynamic Service Discovery. In: IEEE/ACM International Conference on Automated Software Engineering, pp 158-167 (2008) 